\title{
ON THE GEOMETRY OF PETROV TYPE II SPACETIMES
}

\author{
STEFFEN AKSTEINER, LARS ANDERSSON, BERNARDO ARANEDA, AND BERNARD WHITING
}

\begin{abstract}
In general, geometries of Petrov type II do not admit symmetries in terms of Killing vectors or spinors. We introduce a weaker form of Killing equations which do admit solutions. In particular, there is an analog of the Penrose-Walker Killing spinor. Some of its properties, including associated conservation laws, are discussed. Perturbations of Petrov type II Einstein geometries in terms of a complex scalar Debye potential yield complex solutions to the linearized Einstein equations. The complex linearized Weyl tensor is shown to be half Petrov type N. The remaining curvature component on the algebraically special side is reduced to a first order differential operator acting on the potential.
\end{abstract}

\section{INTRODUCTION}

A remarkable property of vacuum spacetimes of Petrov type D is the existence of 'hidden symmetries', namely, appropriate generalizations of Killing vectors such as Killing tensors and conformal Killing-Yano tensors. Penrose and Walker have shown [30] the existence of a valence 2 Killing spinor, from which one can obtain the above mentioned symmetries. Because of the Goldberg-Sachs theorem, the vacuum type D condition is equivalent to the existence of two independent null geodesic congruences that are shear-free. On the other hand, in general, vacuum spacetimes of Petrov type II do not possess any symmetries or hidden symmetries. However they do admit a shear-free null geodesic congruence. In this paper we show that this can be used to define a weaker version of the Killing equations, and we show that they are solved by a generalization of the Penrose-Walker Killing spinor.

The construction of solutions to the field equations for linear spinning fields in terms of scalar, tensorial or spinorial potentials has a long history and has been widely studied. In the case of the Maxwell field, the main names associated to this procedure are Debye and Hertz. Although the usage in the literature is not consistent, see Stewart [26], we shall here refer to scalar potentials of the above mentioned type as Debye potentials. In this paper we focus on the spin- 2 case, and consider the construction of solutions to the linearized Einstein equations on backgrounds of Petrov type II, in terms of Debye potentials. The analogous construction on backgrounds of Petrov type D, including the Schwarzschild and Kerr spacetimes has been widely studied, see e.g. [15, 29, 9], and plays an important role in the study of black hole perturbations [16], 11] and the self-force problem [17], [8]. The construction of solutions to the linearized Einstein equation by the Debye potential method on backgrounds of Petrov type II is possible and is closely analogous to the type D case. Kegeles and Cohen [15] and Stewart [26] have carried out a systematic study of the Debye potential construction in this case, and in particular, Stewart calculated the tetrad components of the linearized Weyl tensor. In this work we show that the linearized Weyl tensor is half type $\mathrm{N} 1$.

It is worth recalling briefly the situation for vacuum type D spacetimes. We shall use the conventions and notation of [18, 19]. A spacetime of Petrov type D admits two repeated principal null directions $l^{a}, n^{a}$, which have corresponding principal spin dyad $o^{A}, \iota^{A}$. In terms of this principal frame, the only non-vanishing Weyl scalar is $\Psi_{2}$. As was shown in [30], vacuum spacetimes (for the more general situation see [4]) of Petrov type D admit a Killing spinor of the form

$$
\stackrel{\circ}{K}_{A B}=\Psi_{2}^{-1 / 3} o_{(A} \iota_{B)}
$$

(the ring "०" here is notation only intended to separate the type D case from the type II case that we discuss below), which satisfies the equation

$$
\nabla_{A^{\prime}(A} \stackrel{\circ}{K}_{B C)}=0 .
$$

\footnotetext{
${ }^{1}$ The linearized metric generated from a Debye potential is naturally complex and therefore there are two Petrov classifications and half types possible, see below for details.
} 
Different kinds of symmetries are associated to the object (1.1), see [12], [19, Section 6.7]. In particular in vacuum, the vector field defined by

$$
\stackrel{\circ}{\xi}_{A A^{\prime}}=\nabla_{A^{\prime}}^{B} \stackrel{\circ}{K}_{A B}
$$

is a Killing vector, $\left.\nabla_{(a} \stackrel{\circ}{\xi}\right)_{b}=0$. In the Kerr-NUT class, from (1.1) one can also construct a Killing tensor $H_{a b}$ and a second Killing vector $\eta_{a}=H_{a b} \stackrel{\circ}{\xi}^{b}$, see [12] for details.

Consider an Einstein spacetime, i.e. the Einstein tensor being proportional to the metric or equivalently vacuum with cosmological constant, of Petrov type II. By [13], a spacetime with a Killing spinor of valence 2, cf. eq. (1.2), has Weyl tensor of type D, N or O, so a type II geometry does not admit a Killing spinor of valence 2. Instead, we prove in section 3 below:

Theorem 1. Let $\left(\mathcal{M}, g_{a b}\right)$ be a real Einstein spacetime of Petrov type II, and let $o_{A}, \iota_{A}$ be a spin dyad, such that $o_{A}$ is a repeated principal spinor. Let $\Psi_{i}$ be the corresponding Weyl scalars. Define

$$
\begin{aligned}
K_{A B} & =\Psi_{2}^{-1 / 3} o_{(A} \iota_{B)}-\frac{1}{3} \Psi_{2}^{-4 / 3} \Psi_{3} o_{A} O_{B}, \\
\xi_{A A^{\prime}} & =\nabla^{B}{ }_{A^{\prime}} K_{A B} .
\end{aligned}
$$

Then

(1) $K_{A B}$ solves the "projected" Killing spinor equation

$$
o^{A} \nabla_{A^{\prime}(A} K_{B C)}=0
$$

(2) $\xi_{A A^{\prime}}$ solves the "projected" Killing equation

$$
o^{A}\left(\nabla_{A A^{\prime}} \xi_{B B^{\prime}}+\nabla_{B B^{\prime}} \xi_{A A^{\prime}}\right)=0 .
$$

Now, the Debye potential construction produces complex solutions to the linearized Einstein equations, which leads to the possibility of having "half types" for the Weyl tensor. Recall first that in a real, four-dimensional orientable manifold with a metric of Lorentzian signature, the Hodge star operator $*$ acting on 2 -forms satisfies $*^{2}=-1$, thus it has eigenvalues $\pm i$. As a consequence, the eigenspaces of $*$ are complex, i.e. a self-dual (SD) or anti-self-dual (ASD) 2form is necessarily complex. Any real 2-form can be written as the sum of a SD part and an ASD part, and these pieces are complex conjugates of each other. Since the Weyl tensor $C_{a b c d}$ can be regarded as a tensor-valued 2-form, a similaRózgar discussion applies to it. Namely, $C_{a b c d}$ can be written as the sum of a SD piece $C_{a b c d}^{+}$and an ASD piece $C_{a b c d}^{-}$, where $C_{a b c d}^{+}$and $C_{a b c d}^{-}$ are complex conjugates of each other. In spinor terms (see section 2 for notation and details), these are $C_{a b c d}^{-}=\Psi_{A B C D} \bar{\epsilon}_{A^{\prime} B^{\prime}} \bar{\epsilon}_{C^{\prime} D^{\prime}}$ and $C_{a b c d}^{+}=\bar{\Psi}_{A^{\prime} B^{\prime} C^{\prime} D^{\prime}} \epsilon_{A B} \epsilon_{C D}$, where $\Psi_{A B C D}$ is the Weyl curvature spinor, see [18, Section 4.6].

On the other hand, if the spacetime metric is complex, the above decomposition still holds, but the pieces $C_{a b c d}^{+}$and $C_{a b c d}^{-}$are now independent entities, so one has two independent Weyl spinors $\Psi_{A B C D}$ and $\tilde{\Psi}_{A^{\prime} B^{\prime} C^{\prime} D^{\prime}}$, see [19, Section 6.9]. In particular, the Petrov types of $\Psi_{A B C D}$ and $\tilde{\Psi}_{A^{\prime} B^{\prime} C^{\prime} D^{\prime}}$ are independent; for example, one part may be algebraically special while the other one is algebraically genera 2 . This also applies to linearized gravity, where even if the background metric is real, a complex perturbation will in general have independent SD and ASD linearized curvatures. In what follows we denote the SD and ASD linearized curvature spinors for a complex perturbation by $\dot{\tilde{\Psi}}_{A^{\prime} B^{\prime} C^{\prime} D^{\prime}}$ and $\dot{\Psi}_{A B C D}$ respectively, see section 2 for details.

We shall make use of the Geroch-Held-Penrose (GHP) formalism 10]. For algebraically special spacetimes, the method of adjoint operators introduced by Wald in [29] (see section 4 for a brief review) can be used to show that if a scalar field $\chi$ of GHP weight $\{-4,0\}$ solves the Debye equation

$$
\left(\left(\mathrm{p}^{\prime}-\bar{\rho}^{\prime}\right)(\mathrm{p}+3 \rho)-\left(\check{\partial}^{\prime}-\bar{\tau}\right)(ð+3 \tau)-3 \Psi_{2}\right) \chi=0
$$

then the complex tensor field $h_{a b}=\mathcal{S}^{\dagger}(\chi)_{a b}$, where $\mathcal{S}^{\dagger}$ is the adjoint of the operator $\mathcal{S}$ defined in eq. 4.2a below, is a solution to the linearized Einstein vacuum equations (possibly with cosmological constant). For perturbations of vacuum type D spacetimes, it was shown in [15]

\footnotetext{
${ }^{2}$ Note however that due to results by Rózga 23], in order for a complex 4-dimensional spacetime to admit a real, Lorentzian slice, the algebraic type of $\Psi_{A B C D}$ and $\tilde{\Psi}_{A B C D}$ must be the same. See also 31 ].
} 
that the ASD linearized Weyl spinor $\dot{\Psi}_{A B C D}$ has special algebraic structure, viz. it is of Petrov type $\mathrm{N}$,

$$
\dot{\Psi}_{A B C D}=o_{A} o_{B} o_{C} o_{D} \dot{\Psi}_{4},
$$

whereas the SD linearized Weyl spinor may be algebraically general.

Furthermore, while the linearized Weyl scalars associated to a Debye potential $\chi$ are given in general by fourth order differential operators applied to $\stackrel{\circ}{\chi}$, it was shown in [15], see also [3], that the scalar field $\dot{\Psi}_{4}$ in (1.9) is given by the simple expression,

$$
\dot{\Psi}_{4}=c \mathcal{L}_{\xi} \dot{\chi},
$$

with (possibly complex) constant $c$. Here, $\mathcal{L}_{\xi}$ is the Lie derivative along $\dot{\xi}^{a}$, which itself is given by (1.5) for the type D Killing spinor. In the Kerr spacetime, (1.10) is essentially the time derivative of $\dot{\chi}$, see also [16]. For Petrov type D, this reduction was done by Kegeles and Cohen [15] for the vacuum case and by Torres del Castillo [27] including a cosmological constant 3 .

One may think that the remarkably simple structure (1.9)-(1.10) is associated to the very special symmetry properties of vacuum type D spacetimes, i.e. to the existence of the "hidden" symmetry (1.1) and the two associated isometries mentioned before. In this note we generalize these results to Petrov type II spacetimes, which in general do not possess any isometries:

Theorem 2. Consider an Einstein spacetime of Petrov type II with repeated principal spinor $o^{A}$. Let $h_{a b}$ be a complex solution to the linearized Einstein vacuum equations generated by a Debye potential $\chi$. Then

(1) the ASD Weyl spinor of $h_{a b}$ is of Petrov type $N$,

$$
\dot{\Psi}_{A B C D}=o_{A} o_{B} o_{C} o_{D} \dot{\Psi}_{4} .
$$

(2) the non-vanishing component of (1.11) is given by

$$
\dot{\Psi}_{4}=-\left(\Psi_{2}^{4 / 3} \xi^{a} \Theta_{a}+3 \Psi_{2}^{2}+6 \Psi_{2} \Lambda\right) \chi .
$$

Here $\xi^{a}$ is given by (1.5), where $K_{A B}$ is the projected Killing spinor (1.4), $\Theta_{a}$ is the GHP connection and $6 \Lambda$ corresponds to the cosmological constant.

Remark 3. Upon finishing this work, we found a virtually unknown preprint by Jeffryes, [14], about half-algebraically special geometries and potentials for field equations. Although Theorem 2 can alternatively be obtained from $\$ 8$ of that work, the interpretation of the derivative in terms of a projected Killing vector has not been given there. In fact the focus of that work was on the non-linear case coupled to Yang-Mills and the relation to the situation here is quite intricate, see remark 39 for further details.

In the case of linearized gravity in Minkowski spacetime, it was shown in [28] that real solutions of the linearized Einstein vacuum equations are in one-to-one correspondence with complex solutions with half-flat curvature, which in turn are in one-to-one correspondence with solutions of the scalar wave equation. The result of Theorem 2 tells us that, while the complex metric perturbation generated by a Debye potential in a type II space is not half-flat, the linearized curvature has a simple structure since it is half type N.

For vacuum type $\mathrm{D}$ spacetimes, it is sometimes assumed that, up to gauge, all real solutions of the linearized Einstein vacuum equations can be obtained, locally, as the real part of a metric generated by a Debye potential; for recent advances in the Schwarzschild and Kerr cases see respectively [20] and [11]. For the more general vacuum type II case, from these considerations we expect the result of Theorem 2 to be of relevance for addressing the following conjecture:

Conjecture. All real solutions of the linearized Einstein vacuum equations on a vacuum type II background can be locally obtained, up to gauge, as the real part of the metric generated by a Debye potential.

We also point out, that Jeffryes in [14] made remarks supporting the validity of this conjecture.

\footnotetext{
${ }^{3}$ One of the Authors, B.W., also did this tedious computation including a cosmological constant in 1983, but did not publish it.
} 
Remark 4. Metrics generated from a Debye potential are always in radiation gauge. It is known that any perturbation of Petrov type II geometries can be transformed into radiation gauge, see [21].

Theorem 2 generalizes the result (1.9)-(1.10) in the type D case to type II. We stress that a generic type II spacetime does not possess any ordinary or hidden symmetries, but only the more general objects (1.4) and (1.5) introduced in this work. While it can be shown that the ordinary, valence-2 Killing spinor equation is equivalent to the real, conformal Killing-Yano equation, which is itself a generalization to differential forms of the conformal Killing equation, for the 'projected' Killing spinor equation (1.6) no such equivalence exists. We will give a geometric interpretation to the origin of (1.6) in terms of spinors that are parallel under a suitable connection especially adapted to the geometry, which is the conformal-GHP connection. This also allows us to generalize the result (1.4)-(1.6) to non-vacuum spacetimes in the real-analytic case, and to derive conservation laws associated to projected Killing spinors.

In appendix A, we also review the Robinson-Trautman reduction of the Einstein equations which admits solutions of various Petrov types. In particular there is a Petrov type II solution which we use in example 18 to compute the projected Killing vector $\xi^{a}$.

Most computations were performed with Spinframes [2], based on the symbolic computer algebra package xAct for Mathematica.

\section{Preliminaries}

2.1. The 2-spinor formalism. In this paper we shall make extensive use of the 2-spinor formalism, following the notation and conventions in [18, 19]. The spinor bundles $\mathbb{S} \rightarrow \mathcal{M}$ and $\mathbb{S}^{\prime} \rightarrow \mathcal{M}$ are rank-2 vector bundles with symplectic forms $\epsilon_{A B}$ and $\bar{\epsilon}_{A^{\prime} B^{\prime}}$, such that $T \mathcal{M} \otimes \mathbb{C} \cong \mathbb{S} \otimes \mathbb{S}^{\prime}$ and $g_{a b}=\epsilon_{A B} \bar{\epsilon}_{A^{\prime} B^{\prime}}$. The spaces of SD and ASD 2-forms are written in spinor terms as $\Lambda_{+}^{2} \cong \mathbb{S}^{\prime *} \odot \mathbb{S}^{\prime *}$ and $\Lambda_{-}^{2} \cong \mathbb{S}^{*} \odot \mathbb{S}^{*}$ (with $\mathbb{S}^{*}$ the dual of $\mathbb{S}$, etc.); in other words, a real 2 -form $F_{a b}=F_{[a b]}$ has the spinor decomposition

$$
F_{a b}=\phi_{A B} \bar{\epsilon}_{A^{\prime} B^{\prime}}+\bar{\phi}_{A^{\prime} B^{\prime}} \epsilon_{A B}
$$

where $F_{a b}^{-}=\phi_{A B} \bar{\epsilon}_{A^{\prime} B^{\prime}}$ and $F_{a b}^{+}=\bar{\phi}_{A^{\prime} B^{\prime}} \epsilon_{A B}$ are the SD and ASD parts of $F_{a b}$ respectively, and $\phi_{A B}=\phi_{(A B)}$. The Riemann tensor admits a similar decomposition [18, Eq. (4.6.38)]:

$$
\begin{aligned}
R_{a b c d}= & \Psi_{A B C D} \bar{\epsilon}_{A^{\prime} B^{\prime}} \bar{\epsilon}_{C^{\prime} D^{\prime}}+\bar{\Psi}_{A^{\prime} B^{\prime} C^{\prime} D^{\prime}} \epsilon_{A B} \epsilon_{C D} \\
& +\Phi_{A B C^{\prime} D^{\prime}} \bar{\epsilon}_{A^{\prime} B^{\prime}} \epsilon_{C D}+\Phi_{A^{\prime} B^{\prime} C D} \epsilon_{A B} \bar{\epsilon}_{C^{\prime} D^{\prime}} \\
& +2 \Lambda\left(\epsilon_{A C} \epsilon_{B D} \bar{\epsilon}_{A^{\prime} C^{\prime}} \bar{\epsilon}_{B^{\prime} D^{\prime}}-\epsilon_{A D} \epsilon_{B C} \bar{\epsilon}_{A^{\prime} D^{\prime}} \bar{\epsilon}_{B^{\prime} C^{\prime}}\right)
\end{aligned}
$$

where $\Psi_{A B C D}=\Psi_{(A B C D)}$ is the Weyl conformal spinor, $\Phi_{A B C^{\prime} D^{\prime}}=\Phi_{(A B)\left(C^{\prime} D^{\prime}\right)}$ is the trace-free Ricci spinor (which is real, i.e. $\bar{\Phi}_{a b}=\Phi_{a b}$ ), and $\Lambda=R / 24$ represents the scalar curvature (which is also real, $\bar{\Lambda}=\Lambda) \sqrt{4}$.

2.2. Self-duality. Let $\left(\mathcal{M}, g_{a b}\right)$ be a real, four-dimensional Lorentzian manifold. Throughout we make use of the abstract index notation. We assume the spacetime to be orientable so that there is a volume form $\varepsilon_{a b c d}$ and the associated Hodge star $*: \Lambda^{k} \rightarrow \Lambda^{4-k}$, where $\Lambda^{k}$ is the space of $k$-forms. For 2 -forms, $*$ satisfies $*^{2}=-1$; this induces a decomposition $\Lambda^{2}=\Lambda_{+}^{2} \oplus \Lambda_{-}^{2}$, where $\Lambda_{+}^{2}$ (resp. $\Lambda_{-}^{2}$ ) is the rank 3 eigenbundle of $*$ associated to the eigenvalue $+i$ (resp. $-i$ ). Elements of $\Lambda_{+}^{2}$ are called self-dual (SD) 2-forms, and those of $\Lambda_{-}^{2}$ are anti-self-dual (ASD) 2-forms. Since the Riemann curvature tensor has the symmetries $R_{a b c d}=R_{[a b][c d]}$, one can also apply the Hodge duality operation to it, in particular this defines the left- and right-dual Riemann tensors by ${ }^{*} R_{a b c d}=\frac{1}{2} \varepsilon_{a b}{ }^{e f} R_{e f c d}$ and $R_{a b c d}^{*}=\frac{1}{2} \varepsilon_{c d}{ }^{e f} R_{a b e f}$, respectively. For the Weyl tensor, the left- and right-duals coincide: ${ }^{*} C_{a b c d}=C_{a b c d}^{*}$. One then defines the SD and ASD Weyl tensors by

$$
C_{a b c d}^{ \pm}:=\frac{1}{2}\left(C_{a b c d} \mp i^{*} C_{a b c d}\right) \text {. }
$$

These tensors satisfy $* C_{a b c d}^{ \pm}= \pm i C_{a b c d}^{ \pm}$.

In spinor terms, the SD and ASD Weyl tensors (2.3) are

$$
C_{a b c d}^{-}=\Psi_{A B C D} \bar{\epsilon}_{A^{\prime} B^{\prime}} \bar{\epsilon}_{C^{\prime} D^{\prime}}, \quad C_{a b c d}^{+}=\bar{\Psi}_{A^{\prime} B^{\prime} C^{\prime} D^{\prime}} \epsilon_{A B} \epsilon_{C D}
$$

\footnotetext{
${ }^{4}$ In the Einstein case, we have $\Phi_{A B C^{\prime} D^{\prime}}=0$ and cosmological constant $6 \Lambda$.
} 
In particular, the SD and ASD Weyl tensors are complex conjugate of each other, which follows from (2.3).

2.3. Complex geometries. For complex geometries, the above decompositions still apply, but now a pair of complex conjugate quantities that appear together in a real expression such as (2.1), is replaced by two independent entities; see [19, Section 6.9]. For example, for a complex 2 -form, the spinor $\bar{\phi}_{A^{\prime} B^{\prime}}$ in (2.1) is replaced by a spinor $\psi_{A^{\prime} B^{\prime}}$ which is no longer the complex conjugate of $\phi_{A B}$. Similarly, the Riemann tensor of a complex metric has a spinor decomposition analogous to (2.2), but where $\bar{\Psi}_{A^{\prime} B^{\prime} C^{\prime} D^{\prime}}$ is now replaced by another spinor $\widetilde{\Psi}_{A^{\prime} B^{\prime} C^{\prime} D^{\prime}}$ which is no longer the complex conjugate of $\Psi_{A B C D}$. The Ricci spinor $\Phi_{A B C^{\prime} D^{\prime}}$ and the scalar curvature $\Lambda$ do not acquire "tilded" versions because of the original reality conditions $\bar{\Phi}_{a b}=\Phi_{a b}$ and $\bar{\Lambda}=\Lambda$, which are a consequence of the symmetries of the Riemann tensor; they simply become complex objects.

The fact that $\Psi_{A B C D}$ and $\widetilde{\Psi}_{A^{\prime} B^{\prime} C^{\prime} D^{\prime}}$ are now two independent entities implies that one correspondingly has two independent algebraic classification schemes for the Weyl curvature spinors, so we can have for example conformally 'half-flat' manifolds if, say, $\Psi_{A B C D}=0$ and $\widetilde{\Psi}_{A^{\prime} B^{\prime} C^{\prime} D^{\prime}} \neq 0$, or 'half-algebraically special' solutions if $\Psi_{A B C D}$ is algebraically special while $\widetilde{\Psi}_{A^{\prime} B^{\prime} C^{\prime} D^{\prime}}$ is general. These remarks about complex geometries apply of course also to complex, linear perturbations of real geometries.

2.4. Conformal-GHP connections. In section 3 we shall discuss some geometric aspects of projected Killing spinors. In order to do this we need an extension of the GHP derivative that includes conformal transformations. We call such an extension the 'conformal-GHP' connection, and give a brief review of some aspects of the construction that are relevant for this work.

Let $\left(\mathcal{M}, g_{a b}\right)$ be a Lorentzian spacetime, with Levi-Civita connection $\nabla_{a}$. Let $\left(o^{A}, \iota^{A}\right)$ be a general spin dyad. We allow two kinds of transformations: (i) 'GHP transformations' $o^{A} \rightarrow \gamma o^{A}$, $\iota^{A} \rightarrow \gamma^{-1} \iota^{A}$, with $\gamma$ a non-vanishing complex scalar field, and (ii) conformal transformations $o^{A} \rightarrow o^{A}, \iota^{A} \rightarrow \Omega^{-1} \iota^{A}$, where $\Omega$ is a positive scalar field. The transformation (ii) is induced by a transformation of the spin metric $\epsilon^{A B} \rightarrow \Omega^{-1} \epsilon^{A B}$, which is in turn induced by a conformal transformation of the metric, $g^{a b} \rightarrow \Omega^{-2} g^{a b}$.

Let $\mathbb{S}_{\{p, q\}}[w]$ be the vector bundle of conformally weighted spinors with GHP weight $\{p, q\}$ and conformal weight $w$, and with an arbitrary index structure. This means that a section $\varphi_{B \ldots B^{\prime} \ldots}^{A \ldots} \in \Gamma\left(\mathbb{S}_{\{p, q\}}[w]\right)$ transforms as $\varphi_{B \ldots B^{\prime} \ldots A^{\prime} \ldots}^{A} \ldots \gamma^{p} \bar{\gamma}^{q} \varphi_{B \ldots}^{A \ldots A^{\prime} \ldots}$ under GHP transformations, and as $\varphi_{B}^{A \ldots A^{\prime} \ldots} \rightarrow \Omega^{w} \varphi_{B \ldots}^{A} \ldots A^{\prime} \ldots$ under conformal transformations. For example, we have $o^{A} \in$ $\Gamma\left(\mathbb{S}_{\{1,0\}}[0]\right)$ and $\iota^{A} \in \Gamma\left(\mathbb{S}_{\{-1,0\}}[-1]\right)$. The conformal-GHP covariant derivative is a linear connection $\mathcal{C}_{A A^{\prime}}: \Gamma\left(\mathbb{S}_{\{p, q\}}[w]\right) \rightarrow \Gamma\left(T^{*} \mathcal{M} \otimes \mathbb{S}_{\{p, q\}}[w]\right)$. In this work all objects considered have vanishing ' $q$-weight', so we shall restrict to quantities of GHP weight $\{p, 0\}$. For the general case and more details see [5] and references therein. The action of $\mathcal{C}_{A A^{\prime}}$ on, say, a spinor field $\varphi_{C C^{\prime}}^{B B^{\prime}}$ with GHP weight $\{p, 0\}$ and conformal weight $w$, is given by

$$
\begin{aligned}
\mathcal{C}_{A A^{\prime}} \varphi_{C C^{\prime}}^{B B^{\prime}}= & \nabla_{A A^{\prime}} \varphi_{C C^{\prime}}^{B B^{\prime}}+\left(w f_{A A^{\prime}}+p\left(\omega_{A A^{\prime}}+B_{A A^{\prime}}\right)\right) \varphi_{C C^{\prime}}^{B B^{\prime}} \\
& +\epsilon_{A}{ }^{B} f_{Q A^{\prime}} \varphi_{C C^{\prime}}^{Q B^{\prime}}+\epsilon_{A^{\prime}}{ }^{B^{\prime}} f_{A Q^{\prime}} \varphi_{C C^{\prime}}^{B Q^{\prime}}-f_{C A^{\prime}} \varphi_{A C^{\prime}}^{B B^{\prime}}-f_{A C^{\prime}} \varphi_{C A^{\prime}}^{B B^{\prime}},
\end{aligned}
$$

with

$$
\begin{aligned}
& \omega_{a}:=-\epsilon n_{a}+\epsilon^{\prime} \ell_{a}-\beta^{\prime} m_{a}+\beta \bar{m}_{a}, \\
& B_{a}:=-\rho n_{a}+\tau \bar{m}_{a}, \\
& f_{a}:=\rho n_{a}+\rho^{\prime} \ell_{a}-\tau^{\prime} m_{a}-\tau \bar{m}_{a},
\end{aligned}
$$

where we are using standard GHP notation for spin coefficients. For spinors with a different index structure, the corresponding action of $\mathcal{C}_{A A^{\prime}}$ can be deduced from (2.5) by linearity and the Leibniz rule. By construction, the connection (2.5) is covariant under combined conformal and GHP transformations,

$$
\mathcal{C}_{a} \varphi_{C \ldots C^{\prime} \ldots}^{B \ldots} \rightarrow \gamma^{p} \Omega^{w} \mathcal{C}_{a} \varphi_{C \ldots C^{\prime} \ldots}^{B} \ldots B^{\prime} \ldots
$$

\footnotetext{
${ }^{5}$ When acting on scalar quantities, the projection of $\mathcal{C}_{A A^{\prime}}$ on a null tetrad reduces to the conformally invariant GHP operators of Penrose and Rindler [18, Eq. (5.6.36)]. We note however that, for general spinors and tensors, the operators in [18, Eq. (5.6.36)] do not map conformal densities to conformal densities.
} 
In particular, applying (2.5) to the spin frame $\left(o_{A}, \iota_{A}\right)$ and taking into account that $o_{A} \in$ $\Gamma\left(\mathbb{S}_{\{1,0\}}[1]\right)$ and $\iota_{A} \in \Gamma\left(\mathbb{S}_{\{-1,0\}}[0]\right)$, one finds

$$
\begin{aligned}
& \mathcal{C}_{A A^{\prime} o_{B}}=\left(o^{C} o^{D} \nabla_{C A^{\prime} o_{D}}\right) \iota_{A} \iota_{B}, \\
& \mathcal{C}_{A A^{\prime} \iota_{B}}=\left(\iota^{C} \iota^{D} \nabla_{C A^{\prime} \iota_{D}}\right) o_{A} o_{B} .
\end{aligned}
$$

Remark 5. Analogously to the usual GHP connection, the conformal-GHP connection $\mathcal{C}_{A A^{\prime}}$ depends on the choice of a spin dyad $\left(o^{A}, \iota^{A}\right)$. In what follows, the dyad $\left(o^{A}, \iota^{A}\right)$ will always be understood to be the one associated to $\mathcal{C}_{A A^{\prime}}$.

\section{SPECial GeOMetry AND PARALlel SPINORS}

In this section we provide a geometric interpretation for projected Killing spinors and discuss general properties such as integrability conditions, the solution space and a relation to conservation laws. Except for some specific examples, we do not assume the geometry to be Ricci-flat. Several results below need a complexification of spacetime and in those situations we impose real-analyticity; this will be explicitly indicated in each case.

We will first show that the projected Killing spinor equation can be understood as a consequence of the existence of spinors that are parallel under the conformal-GHP connection.

Remark 6. The formulation in this section is conformally invariant (except for the specific examples in which we assume the Einstein condition, see (2) in Lemma 12 and examples 17 and 22). This means that all results below are valid not only for a specific metric but for the equivalence class of metrics conformally related to each other, i.e. for conformal structures. For simplicity, however, we shall state the results in terms of spacetimes and not conformal structures.

Let $\left(\mathcal{M}, g_{a b}\right)$ be a Lorentzian spacetime, and let $o^{A}$ be a spinor field satisfying

$$
o^{A} O^{B} \nabla_{A A^{\prime} O_{B}}=0 .
$$

Such an $o^{A}$ will be called a shear-free ray (SFR). If $o_{A}$ is SFR, it follows from (2.7a) that it is parallel under $\mathcal{C}_{A A^{\prime}}$. In GHP notation, (3.1) is equivalent to $\kappa=\sigma=0$. The condition $\kappa=0$ encodes that the null congruence associated to the vector field $o^{A} \bar{o}^{A^{\prime}}$ is geodesic, whereas $\sigma=0$ states that this congruence is shear-free. Thus, a non-trivial solution to (3.1) imposes the existence of a shear-free null geodesic congruence.

Remark 7. Several results below require a complexified spacetime, for which we need to impose real-analyticity. The complex extension of $\mathcal{M}$ will be denoted $\mathbb{C} \mathcal{M}$. Our interest here is in the case where $\mathbb{C M}$ arises as the complexification of a real spacetime with Lorentzian signature, see [19, Section 6.9].

The reason why we need to complexify $\mathcal{M}$ is the following important result about SFRs:

Lemma 8 (Proposition (7.3.18) in [19]). Let $o^{A}$ be a spinor, and let $\mu^{A^{\prime}}, \nu^{A^{\prime}}$ be a primed spin dyad. Then the complex vector fields $X^{a}=o^{A} \mu^{A^{\prime}}, Y^{a}=o^{A} \nu^{A^{\prime}}$ on $\mathbb{C N}$ are in involution if and only if $o^{A}$ is SFR.

Hence by Frobenius' theorem, the distribution defined by $\left\{X^{a}, Y^{a}\right\}$ is surface-forming in $\mathbb{C} \mathcal{M}$. The surfaces associated to this distribution are complex, and they are called $\beta$-surfaces, see e.g. [19, pp. 309-310]:

Definition 9 ( $\beta$-surface). A $\beta$-surface is a complex 2-dimensional surface in $\mathbb{C} \mathcal{M}$ whose tangent vectors at any one point are all of the form $o^{A} \mu^{A^{\prime}}$ for some $\mu^{A^{\prime}}$, where $o^{A}$ is fixed and satisfies equation (3.1).

For the following it is convenient to introduce some additional notation.

Definition 10. Let $\mathcal{C}_{A A^{\prime}}$ be the conformal-GHP connection associated to a spin dyad $\left(o^{A}, \iota^{A}\right)$. We define the operators

$$
\tilde{\mathcal{C}}_{A^{\prime}}:=o^{A} \mathcal{C}_{A A^{\prime}}, \quad \mathcal{C}_{A^{\prime}}:=\iota^{A} \mathcal{C}_{A A^{\prime}}
$$


It is worth discussing some properties of $\tilde{\mathcal{C}}_{A^{\prime}}$. It is a linear operator that satisfies the Leibniz rule, and which maps a section of $\mathbb{S}_{\{p, 0\}}[w]$ into a section of $\mathbb{S}^{\prime} \otimes \mathbb{S}_{\{p+1,0\}}[w]$, where $\mathbb{S}^{\prime}$ is the primed spin bundle introduced in section 2, Consider now a $\beta$-surface $\Sigma$ in $\mathbb{C} \mathcal{M}$. Any element of the tangent bundle $T \Sigma$ is of the form $\left(x, o^{A} \mu^{A^{\prime}}\right)$, where $x \in \Sigma, o^{A}$ is fixed, and $\mu^{A^{\prime}}$ is some primed spinor at $x$. Therefore we can identify $T \Sigma$ with the restriction of the primed spin bundle to $\Sigma,\left.\mathbb{S}^{\prime}\right|_{\Sigma}$. Similarly, the cotangent bundle $T^{*} \Sigma$ can be identified with the dual $\left.\mathbb{S}^{\prime *}\right|_{\Sigma}$. This means that a primed spinor field $\psi_{A^{\prime}}$ can be thought of as a "1-form" in $T^{*} \Sigma$. Therefore, restricting to $\beta$-surfaces, the operator $\tilde{\mathcal{C}}_{A^{\prime}}$ is $\tilde{\mathcal{C}}_{A^{\prime}}: \Gamma\left(\mathbb{S}_{\{p, 0\}}[w]\right) \rightarrow \Gamma\left(T^{*} \Sigma \otimes \mathbb{S}_{\{p+1,0\}}[w]\right)$, from which we see that it can be regarded as a connection, in the usual sense, on conformally and GHP-weighted vector bundles over $\beta$-surfaces on $\mathbb{C} \mathcal{M}$, see [6] .

We have the following result, which does not require analyticity:

Lemma 11. Let $\left(\mathcal{M}, g_{a b}\right)$ be a Lorentzian spacetime with $\tilde{\mathcal{C}}_{A^{\prime}}$ given by (3.2), where $o^{A}$ satisfies (3.1). Then

$$
\left[\tilde{\mathrm{C}}_{A^{\prime}}, \tilde{\mathrm{C}}_{B^{\prime}}\right]=0
$$

if and only if $o^{A}$ is a repeated principal spinor of $\Psi_{A B C D}$, that is

$$
\Psi_{A B C D} O^{B} O^{C} O^{D}=0 .
$$

This result follows from Lemmas 3.4 and 3.5 in [6]. As mentioned, the proof of Lemma 11 does not require analyticity. However, from the discussion above we know that on $\beta$-surfaces, which live in $\mathbb{C} \mathcal{M}$, we can interpret $\tilde{\mathcal{C}}_{A^{\prime}}$ as a connection. In that case the commutator $\left[\tilde{\mathcal{C}}_{A^{\prime}}, \tilde{\mathfrak{C}}_{B^{\prime}}\right]$ is the curvature of $\tilde{\mathcal{C}}_{A^{\prime}}$. Thus, the result of Lemma 11 tells us that, as long as conditions (3.1) and (3.4) hold, $\tilde{\mathfrak{C}}_{A^{\prime}}$ is a flat connection on $\beta$-surfaces. From [6, eq. (3.27)] we see that one can associate a (twisted) de Rham complex to $\tilde{\mathcal{C}}_{A^{\prime}}$. Local exactness of a de Rham complex allows to find (local) potentials in specific situations; for example, we find the following:

Lemma 12. Suppose that $o^{A}$ is SFR (3.1) and a repeated principal spinor (3.4).

(1) Assume $\left(\mathcal{M}, g_{a b}\right)$ is real-analytic with complexification $\mathbb{C} \mathcal{M}$. Then there exist scalar fields $\phi$ and $\eta$ on $\mathbb{C M}$, whose GHP and conformal weights are $\{0,0\}, w=-1$ and $\{-2,0\}$, $w=-1$ respectively, such that $f_{a}$, given in (2.6c), takes the form

$$
f_{A A^{\prime}}=\nabla_{A A^{\prime}} \log \phi-o_{A} \tilde{\mathrm{e}}_{A^{\prime}} \eta \text {. }
$$

(2) Assume that $\left(\mathcal{M}, g_{a b}\right)$ is real and that $g_{a b}$ is Einstein. Then, with Weyl scalars $\Psi_{i}$, (3.5) holds for $\phi$ and $\eta$ given by

$$
\phi=\Psi_{2}^{1 / 3}, \quad \eta=\frac{1}{3} \Psi_{2}^{-1} \Psi_{3} .
$$

The proof of eq. (3.5), along with other results and applications, will be given in a forthcoming publication. This proof involves the existence of $\beta$-surfaces and that is the reason why it is formulated in the real-analytic setting. On the other hand, (3.6) can be easily demonstrated in any Einstein spacetime, not necessarily analytic, by using the GHP form of the Bianchi identities 6 .

Remark 13. Consider the complexified spacetime $\mathbb{C} \mathcal{M}$.

- Since $\tilde{\mathcal{C}}_{A^{\prime}}$ is a flat connection, the equation $\tilde{\mathcal{C}}_{A^{\prime}} \lambda=0$ has non-trivial solutions for any weights $\{p, 0\}$ and $w$. In particular, from (2.5) we see that, choosing $w=p=-1$, we have

$$
0=\tilde{\mathcal{C}}_{A^{\prime}} \lambda=o^{A} \partial_{A A^{\prime}} \lambda-o^{A} \omega_{A A^{\prime}} \lambda,
$$

where we used that $o^{A} f_{A A^{\prime}}=-o^{A} B_{A A^{\prime}}$. It then follows that such $\lambda$ satisfies

$$
o^{A} \partial_{A A^{\prime}} \log \lambda=o^{A} \omega_{A A^{\prime}} .
$$

- Let $\psi$ be a scalar field on $\mathbb{C} \mathcal{M}$ with $G H P$ weight $\{p, 0\}$ and conformal weight $w$. Using (2.5), (3.5) and (3.8), we see that the action of $\tilde{\mathrm{C}}_{A^{\prime}}$ reduces to

$$
\tilde{\mathfrak{C}}_{A^{\prime}} \psi=\phi^{-(w-p)} \lambda^{-p} o^{A} \partial_{A A^{\prime}}\left[\phi^{w-p} \lambda^{p} \psi\right] .
$$

\footnotetext{
${ }^{6}$ Since Bianchi identities are not conformally invariant, the expression (3.6) breaks conformal invariance.
} 
Using formula (3.9), it follows that if the weighted scalar field $\psi$ satisfies $\tilde{\complement}_{A^{\prime}} \psi=0$, then defining $F=\phi^{w-p} \lambda^{p} \psi$, where $\phi$ and $\lambda$ are defined in (3.5) and (3.8) respectively, this is equivalent to

$$
o^{A} \partial_{A A^{\prime}} F=0 \text {. }
$$

On $\mathbb{C} \mathcal{M}$, these functions are said to be 'constant on $\beta$-surfaces', since the operator $o^{A} \partial_{A A^{\prime}}$ represents translations along the $\beta$-surfaces associated to $o^{A}$. That is to say, there exist linearly independent spinor fields $\mu^{A^{\prime}}, \nu^{A^{\prime}}$ such that $o^{A} \mu^{A^{\prime}} \partial_{A A^{\prime}} \equiv \partial_{\tilde{z}}$ and $o^{A} \nu^{A^{\prime}} \partial_{A A^{\prime}} \equiv \partial_{\tilde{w}}$, where $\tilde{z}, \tilde{w}$ are coordinates along the $\beta$-surfaces. Then equation (3.10) can be interpreted as

$$
F=F(z, w),
$$

where $z, w$ are coordinates constant on each $\beta$-surface. Notice that (3.11) is a function on $\mathbb{C} \mathcal{M}$.

Remark 14. Let $\iota^{A}$ be any spinor field independent of $o^{A}$. Then the equation

$$
\iota^{A} \partial_{A A^{\prime}} f=0
$$

has only constant functions as solutions. To see this, note that if (3.12) holds we must also have $\left[\iota^{A} \nabla_{A A^{\prime}}, \iota^{B} \nabla_{B B^{\prime}}\right] f=0$, but

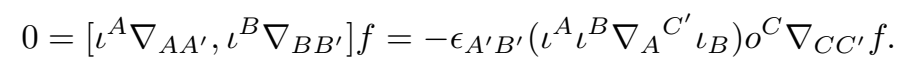

By definition we know that $\iota^{A} \iota^{B} \nabla_{A} C^{C^{\prime}} \iota_{B}=0$ if and only if $\iota^{A}$ is SFR, but this is not the case since $\iota^{A}$ is arbitrary, so we get $o^{C} \nabla_{C C^{\prime}} f=0$, which, when combined with (3.12), implies $\nabla_{A A^{\prime}} f=0$, i.e. $f$ is constant.

In terms of this geometric setup, the projected Killing spinor equation can be deduced as follows:

Proposition 15. Let $\left(\mathcal{M}, g_{a b}\right)$ be a Lorentzian spacetime. Let $\left(o_{A}, \iota_{A}\right)$ be the dyad associated to $\mathrm{C}_{A A^{\prime}}$ (cf. Remark 5), and suppose that $o_{A}$ is SFR.

(1) $o_{A}$ and $\iota_{A}$ are parallel spinors for (3.2):

$$
\tilde{\mathcal{C}}_{A^{\prime} O_{B}}=0, \quad \tilde{\mathcal{C}}_{A^{\prime} \iota_{B}}=0 .
$$

(2) Suppose in addition that $\left(\mathcal{M}, g_{a b}\right)$ is real-analytic and that $o^{A}$ is also a repeated principal spinor, so that eq. (3.5) holds. Then the spinor field

$$
\left.K_{A B}=\phi^{-1}\left[o_{(A} \iota_{B}\right)-\eta o_{A} o_{B}\right]
$$

satisfies the projected Killing spinor equation,

$$
o^{A} \nabla_{A^{\prime}(A} K_{B C)}=0 \text {. }
$$

Proof. Equation (3.14) follows simply from (2.7a) - 2.7b), so we see that analyticity is not required for this item. For the second item, applying the definition (2.5) to the field $o_{B} \iota_{C}$, whose weights are $\{0,0\}$ and $w=1$, we have

$$
\mathcal{C}_{A^{\prime} A}\left(o_{B} \iota_{C}\right)=\nabla_{A^{\prime} A}\left(o_{B} \iota_{C}\right)+f_{A^{\prime} A^{o}{ }_{B} \iota_{C}}-f_{A^{\prime} B} o_{A} \iota_{C}-f_{A^{\prime} C} o_{B} \iota_{A} .
$$

But from 2.7a $-2.7 \mathrm{~b}$ ) we see that

$$
\mathcal{C}_{A^{\prime} A}\left(o_{B} \iota_{C}\right)=\sigma_{A^{\prime}}^{\prime} o_{A} o_{B} o_{C},
$$

thus $\mathcal{C}_{A^{\prime} A}\left(o_{B} \iota_{C}\right)=\mathcal{C}_{A^{\prime}(A}\left(o_{B} \iota_{C}\right)$. Combining with (3.17), we get

$$
\left.\mathcal{C}_{A^{\prime} A}\left(o_{B} \iota_{C}\right)=\mathcal{C}_{A^{\prime}(A}\left(o_{B} \iota_{C}\right)=\left(\nabla_{A^{\prime}(A}-f_{A^{\prime}(A}\right) o_{B} \iota_{C}\right) .
$$

Using (3.5), this is

$$
\begin{aligned}
\mathcal{C}_{A^{\prime} A}\left(o_{B} \iota_{C}\right) & \left.=\phi \nabla_{A^{\prime}(A}\left[\phi^{-1} o_{B} \iota_{C}\right)\right]+o_{(A} o_{B} \iota_{C)} \tilde{\mathcal{C}}_{A^{\prime}} \eta \\
& \left.=\phi\left[\nabla_{A^{\prime}(A}\left[\phi^{-1} o_{B} \iota_{C}\right)\right]+\iota_{(A} \tilde{\mathrm{C}}_{\left|A^{\prime}\right|}\left(\phi^{-1} \eta o_{B} o_{C}\right)\right],
\end{aligned}
$$

where in the second line we used that $\tilde{\mathcal{C}}_{A^{\prime} o_{B}}=0, \tilde{\mathcal{C}}_{A^{\prime}} \phi=0$. Contracting this equation with $o^{A}$ and using (3.18) gives

$$
0=o^{A} \nabla_{A^{\prime}(A}\left[\phi^{-1} o_{B} \iota_{C)}\right]+o^{A} \iota_{(A} \tilde{\mathrm{e}}_{\left|A^{\prime}\right|}\left(\phi^{-1} \eta o_{B} o_{C)}\right) .
$$

Let us compute the second term in the right hand side. Using $o^{A} \tilde{\mathcal{C}}_{A^{\prime}}(\cdot)=\tilde{\mathcal{C}}_{A^{\prime}}\left(o^{A} \cdot\right)$, we get

$$
o^{A} \iota_{(A} \tilde{\mathrm{C}}_{\left|A^{\prime}\right|}\left(\phi^{-1} \eta o_{B} o_{C)}\right)=-\frac{1}{3} \tilde{\mathrm{C}}_{A^{\prime}}\left(\phi^{-1} \eta o_{B} o_{C}\right)=-o^{A} \mathcal{C}_{A^{\prime}(A}\left[\phi^{-1} \eta o_{B} o_{C)}\right]
$$


Noticing that the weights of the field $\phi^{-1} \eta o_{B} o_{C}$ are $\{0,0\}$ and $w=2$, and using the definition (2.5), we have

Therefore

$$
\mathcal{C}_{A^{\prime}(A}\left[\phi^{-1} \eta o_{B} o_{C)}\right]=\nabla_{A^{\prime}(A}\left[\phi^{-1} \eta o_{B} o_{C)}\right] .
$$

and thus

hence the result follows.

$$
o^{A} \iota_{(A} \tilde{\mathcal{C}}_{\left|A^{\prime}\right|}\left(\phi^{-1} \eta o_{B} o_{C)}\right)=-o^{A} \nabla_{A^{\prime}(A}\left(\phi^{-1} \eta o_{B} o_{C}\right)
$$

$$
\left.\left.0=o^{A} \nabla_{A^{\prime}(A}\left[\phi^{-1} o_{B} \iota_{C}\right)-\phi^{-1} \eta o_{B} o_{C}\right)\right],
$$

Remark 16. Any spinor field of the form $\omega_{A}=f o_{A}$, where $f$ is of type $\{-1,0\}, w=0$, and satisfies $\tilde{\mathcal{C}}_{A^{\prime}} f=0$, is a solution to the "projected twistor equation"

$$
o^{A} \nabla_{A^{\prime}(A} \omega_{B)}=0 .
$$

This follows from the identity $o^{A} \mathcal{C}_{A^{\prime}\left({ }_{A} \omega_{B)}\right.}=o^{A} \nabla_{A^{\prime}\left(A_{A} \omega_{B)}\right.}$ replacing $\omega_{A}=f o_{A}$.

From Proposition 15 we have that the spinor field (3.15) is a projected Killing spinor in the general class of spacetimes where $o_{A}$ is SFR and a repeated PND but not necessarily Einstein, although in the non-Einstein case it must be real-analytic. This includes, in particular, the Kerr-(A)dS and (analytic) Kerr-Newman-(A)dS spacetimes, where $K_{A B}$ satisfies the usual, i.e. non-projected, Killing spinor equation.

Example 17. Suppose that $\left(\mathcal{M}, g_{a b}\right)$ is Einstein, i.e. $g_{a b}$ satisfies the vacuum Einstein equations with cosmological constant. Then, using (3.6), we find that (3.15) reduces to the expression (1.4) given in the introduction. Furthermore, the vector field

$$
\xi_{A A^{\prime}}=\nabla_{A^{\prime}}^{B} K_{A B}
$$

solve the projected Killing equation

$$
o^{A}\left(\nabla_{A A^{\prime}} \xi_{B B^{\prime}}+\nabla_{B B^{\prime}} \xi_{A A^{\prime}}\right)=0 .
$$

In GHP notation, (3.24) takes the form

$$
\xi_{a}=\frac{3}{2 \Psi_{2}^{1 / 3}}\left(\rho^{\prime} l_{a}-\rho n_{a}-\tau^{\prime} m_{a}+\tau \bar{m}_{a}\right)+\frac{(ð-4 \tau) \Psi_{3}}{2 \Psi_{2}^{4 / 3}} l_{a}-\frac{(b-4 \rho) \Psi_{3}}{2 \Psi_{2}^{4 / 3}} m_{a},
$$

and from the Bianchi identities it follows that

$$
\xi^{a} \nabla_{a} \Psi_{2}=0 .
$$

To give an explicit example, we review the Robinson-Trautman geometries in appendix A. There are known solutions of various Petrov types in this class and in particular there is one of Petrov type II.

Example 18. For the type II Robinson-Trautman solution given in (A.10), the vector (3.26) is of the form

$$
\xi^{a}=\frac{-2(-1)^{2 / 3}}{3 m^{4 / 3}}\left((\zeta+\bar{\zeta})^{3}\left(\partial_{\zeta}\right)^{a}+m\left(\partial_{u}\right)^{a}\right) .
$$

We note that it does not reduce to $\partial_{u}$, which is a Killing vector of that solution.

Let us now discuss the space of projected Killing spinors. In type D spacetimes one can show that the space of valence-2 Killing spinors (1.1) is 1-dimensional, see e.g. [13]. For type II spacetimes, we first show the following:

Proposition 19. Let $\left(\mathcal{M}, g_{a b}\right)$ be a Lorentzian spacetime, where $o^{A}$ is SFR and a repeated principal spinor. Let

$$
K_{A B}=K_{2} o_{A} o_{B}-2 K_{1} o_{(A} \iota_{B}+K_{0} \iota_{A} \iota_{B}
$$

be a projected Killing spinor, i.e. solving (1.6). Then the components satisfy

$$
\begin{aligned}
K_{0} & =0, \\
\tilde{\mathcal{C}}_{A^{\prime}} K_{1} & =0, \\
\tilde{\mathcal{C}}_{A^{\prime}} K_{2}+2 \mathcal{C}_{A^{\prime}} K_{1} & =0 .
\end{aligned}
$$


Proof. We first derive integrability conditions for (1.6) by introducing

$$
Q_{B C A^{\prime}}=o^{A} \nabla_{A^{\prime}(A} K_{B C)} .
$$

For any geometry satisfying (3.1) we find

$$
\Theta_{(A} A^{A^{\prime}} Q_{B C) A^{\prime}}=-4\left(\Psi_{3} K_{0}-\Psi_{1} K_{2}\right) o_{\left(A^{o} B^{\iota}{ }_{C}\right)}+4\left(\Psi_{2} K_{0}-\frac{2}{3} \Psi_{1} K_{1}\right) o_{\left(A^{\iota} \iota^{\iota} C\right)}-2 \Psi_{1} K_{0} \iota_{A} \iota_{B} \iota_{C} .
$$

The integrability conditions are then obtained by setting $Q_{B C A^{\prime}}=0$, so the left hand side of (3.32) vanishes. If $\Psi_{1} \neq 0$, it follows that $K_{A B}=0$ is the only solution to (1.6), so we need to have at least $\Psi_{1}=0$ to have non-trivial solutions. Since we are interested in type II, we can impose $\Psi_{1}=0$. In that case (3.32) leads to the restrictions

$$
\Psi_{3} K_{0}=0, \quad \Psi_{2} K_{0}=0 .
$$

If $K_{0} \neq 0$, the Weyl spinor would be of Petrov type N (i.e. only $\Psi_{4} \neq 0$ ). In that case (3.32) yields no restrictions on $K_{A B}$. For Petrov type D or II, we have $\Psi_{2} \neq 0$ which forces $K_{0}=0$. For $K_{1}$ and $K_{2}$, a compact form of the equations to be satisfied can be obtained in terms of the covariant derivative $\mathcal{C}_{A A^{\prime}}$ and its projections $\tilde{\mathcal{C}}_{A^{\prime}}, \mathcal{C}_{A^{\prime}}$ given in (3.2). Using $K_{0}=0$, and requiring $K_{A B}$ to have conformal weight 2 so that the projected Killing spinor equation is conformally invariant, eq. (1.6) is

$$
0=\tilde{\mathcal{C}}_{A^{\prime}} K_{B C}+o_{B} \mathfrak{C}_{A^{\prime} C} K_{1}+o_{C} \mathfrak{e}_{A^{\prime} B} K_{1} .
$$

Contraction with $o^{B}$ and with $\iota^{B} \iota^{C}$ leads to,

$$
\begin{aligned}
\tilde{\mathfrak{C}}_{A^{\prime}} K_{1} & =0, \\
\tilde{\mathcal{C}}_{A^{\prime}} K_{2}+2 \mathcal{C}_{A^{\prime}} K_{1} & =0,
\end{aligned}
$$

or in GHP notation

$$
\begin{aligned}
(ð+\tau) K_{1}=0, & (\mathrm{~b}+\rho) K_{1}=0, \\
2\left(\mathrm{~b}^{\prime}+\rho^{\prime}\right) K_{1}+(\widetilde{\partial}+2 \tau) K_{2}=0, & 2\left(\partial^{\prime}+\tau^{\prime}\right) K_{1}+(\mathrm{b}+2 \rho) K_{2}=0 .
\end{aligned}
$$

Remark 20 (Solution space of the projected Killing spinor equation). In the real-analytic case, we see from eqs. (3.30b)-(3.30c) that the space of solutions to the projected Killing spinor equation is infinite dimensional. For example, one solution is given in (3.15), but we also see that, taking $K_{1} \equiv 0$, equations (3.30b)-(3.30c) reduce to $\tilde{\mathcal{C}}_{A^{\prime}} K_{2}=0$. It follows from equations (3.9), (3.10) and (3.11) that there are infinitely many solutions to this equation. Any function of the form $F=\lambda^{-2} \phi^{2} K_{2}$, see Remark 13 and Lemma 11 for the definition of $\lambda$ and $\phi$, which is constant on $\beta$-surfaces leads to a projected Killing spinor $K_{A B}=\lambda^{2} \phi^{-2} \mathrm{Fo}_{A} o_{B}$. We also mention that one could in principle consider $K_{1} \neq 0$ and $K_{2} \equiv 0$ (or $\tilde{\mathcal{C}}_{A^{\prime}} K_{2}=0$ ), but then (3.30b)-(3.30c) leads to $\mathcal{C}_{A A^{\prime}} K_{1}=0$, which, taking into account that the weights of $K_{1}$ are $p=0$ and $w=1$, is explicitly $\nabla_{a} K_{1}+f_{a} K_{1}=0$. This implies $f_{a}=-\nabla_{a} \log K_{1}$ and so $\nabla_{[a} f_{b]}=0$, which is a restriction on the geometry: this is satisfied for vacuum type D but not for arbitrary type II spacetimes.

Finally, an interesting property of projected Killing spinors is that they give rise to solutions of the vacuum Maxwell equations, and therefore to conservation laws:

Lemma 21. Let $\left(\mathcal{M}, g_{a b}\right)$ be real-analytic, and let $o^{A}$ be SFR and a repeated principal spinor. Let $K_{A B}$ be a projected Killing spinor as in (3.29). Assume $K_{1}=c \phi^{-1}$, where $\phi$ was introduced in (3.5) and $c$ is an arbitrary constant, possibly zero. Then the spinor field

$$
\varphi_{A B}=-2 \phi^{3} K_{A B}
$$

is a solution to the vacuum Maxwell equations.

The result of this Lemma is valid regardless of the Einstein condition, but it imposes the realanalyticity restriction, because we need the existence of the scalar field $\phi$ introduced in Lemma 12 . If the Einstein condition is assumed, then real-analyticity is not needed, see Example 22 below. 
Proof of Lemma 21, The proof is straightforward in terms of the conformal connection (2.5). Since the field (3.37) has GHP weight $\{0,0\}$ and conformal weight $w=-1$, it follows from (2.5) that $\nabla_{A^{\prime}}{ }^{A} \varphi_{A B}=\mathcal{C}_{A^{\prime}}{ }^{A} \varphi_{A B}$. Replacing expression (3.29) for $K_{A B}$ with $K_{0}=0$, and using $\mathrm{C}_{A A^{\prime} O_{B}}=0$, we get

$$
\begin{aligned}
-\frac{1}{2} \nabla_{A^{\prime}}{ }^{A} \varphi_{A B} & =-o_{B} \tilde{\mathcal{C}}_{A^{\prime}}\left(\phi^{3} K_{2}\right)+\tilde{\mathcal{C}}_{A^{\prime}}\left(\phi^{3} K_{1} \iota_{B}\right)-o_{B} \mathcal{C}_{A^{\prime}}{ }^{A}\left(\phi^{3} K_{1} \iota_{A}\right) \\
& =-o_{B} \phi^{3} \tilde{\mathfrak{C}}_{A^{\prime}} K_{2}+o_{B} \mathcal{C}_{A^{\prime}}\left(\phi^{3} K_{1}\right) \\
& =2 o_{B} \phi^{3} \mathcal{C}_{A^{\prime}} K_{1}+o_{B} \mathcal{C}_{A^{\prime}}\left(\phi^{3} K_{1}\right) \\
& =3 o_{B} \phi^{3} \mathcal{C}_{A^{\prime}} K_{1}+o_{B} K_{1} \mathcal{C}_{A^{\prime}} \phi^{3} \\
& =3 o_{B} \phi^{2} \mathfrak{C}_{A^{\prime}}\left(\phi K_{1}\right)
\end{aligned}
$$

where in the second line we used $0=\tilde{\mathcal{C}}_{A^{\prime}} \phi=\tilde{\mathfrak{C}}_{A^{\prime}} K_{1}=\tilde{\mathcal{C}}_{A^{\prime}} \iota_{B}$ and also $\mathcal{C}_{A^{\prime}}{ }^{A} \iota_{A}=0$ (which follows from (2.7b) ), and in the third line we used eq. (3.30c). Now, the scalar $\phi K_{1}$ has weights $\{0,0\}$ and $w=0$, so from the definition (2.5) we have $\mathcal{C}_{A^{\prime}}\left(\phi K_{1}\right)=\iota^{A} \nabla_{A A^{\prime}}\left(\phi K_{1}\right)$. Therefore $\nabla_{A^{\prime}}{ }^{A} \varphi_{A B}=0$ if and only if $\iota^{A} \nabla_{A A^{\prime}}\left(\phi K_{1}\right)=0$. Since we are assuming a generic type II spacetime with SFR $o^{A}$, this is true if and only if $K_{1}=c \phi^{-1}$ for some constant $c$, which can be zero. This follows from the discussion in Remark 14.

The result of Lemma 21 tells us that, as long as $K_{1}=c \phi^{-1}$, we have the conservation law

$$
\mathrm{d} \mathcal{F}=0, \quad \mathcal{F}_{a b}=\varphi_{A B} \epsilon_{A^{\prime} B^{\prime}} .
$$

We note that, choosing $c=0$, the Maxwell field (3.37) is $\varphi_{A B}=-2 \phi^{3} K_{2} o_{A} o_{B}$, with $\tilde{\mathcal{C}}_{A^{\prime}}\left(\phi^{3} K_{2}\right)=$ 0 . These are the Robinson null fields discussed for example in [19, Theorem 7.3.14].

Example 22 (Spin lowering). If we assume that $\left(\mathcal{M}, g_{a b}\right)$ is Einstein, then from (3.6) we know that $\phi=\Psi_{2}^{1 / 3}$ and we do not need to restrict to real-analytic spacetimes. The Maxwell field (3.37) is $\varphi_{A B}=-2 \Psi_{2} K_{A B}$. Choosing $c=1$, it follows that

$$
\varphi_{A B}=\Psi_{A B C D} K^{C D} .
$$

Thus, in this case, the result of Lemma 21 can be interpreted as a form of Penrose's spin lowering:

$$
\nabla^{A A^{\prime}} \varphi_{A B}=\left(\nabla^{A A^{\prime}} \Psi_{A B C D}\right) K^{C D}+\Psi_{A B C D}\left(\nabla^{A^{\prime}(A} K^{C D)}\right)=0
$$

where the first term on the right vanishes since $\nabla^{A A^{\prime}} \Psi_{A B C D}=0$ are the Bianchi identities for an Einstein space, and the vanishing of the second term follows from the fact that the projected Killing spinor equation (1.6) implies $\nabla^{A^{\prime}\left({ }^{A} K^{C D)}\right.}=A^{A^{\prime}} o^{A} O^{C} o^{D}$ for some $A^{A^{\prime}}$, and then one uses the type II condition (3.4).

Example 23 (Sachs' conservation law). In [24, eq.(5.23)], Sachs found a conservation law for vacuum type II spacetimes, where he chose a tetrad rotation such that $\Psi_{3}=0$ to derive the conservation law

$$
\mathrm{d}\left(\Psi_{2}^{2 / 3} o_{\left(A \iota_{B}\right)} \bar{\epsilon}_{A^{\prime} B^{\prime}}\right)=0 .
$$

From Example 22 we see that this conservation law can be interpreted as a form of Penrose's spin lowering without the necessity of imposing the tetrad gauge $\Psi_{3}=0$, since the components of the Maxwell field (3.40) are

$$
\varphi_{0}=0, \quad \varphi_{1}=\Psi_{2}^{2 / 3}, \quad \varphi_{2}=\frac{2 \Psi_{3}}{3 \Psi_{2}^{1 / 3}},
$$

which, using (3.39), shows the equivalence to the result of Sachs.

\section{Perturbation theory in terms of a Debye potential}

In this section we review first order perturbation theory of Einstein spacetimes of Petrov type II in terms of scalar potentials and prove Theorem 2, We make use of the adjoint operator method introduced by Wald in 29] to generate solutions to the linearized Einstein equations from solutions of a scalar wave-like equation. 
First, recall the expression for the linearized ASD Weyl spinor $\dot{\Psi}_{A B C D}$ in terms of the linearized metric for an Einstein background with metric $g_{a b}$, as given by Penrose and Rindler in [18, Eq. $(5.7 .15)]$,

$$
\dot{\Psi}_{A B C D}=\frac{1}{2} \nabla_{(A}{ }^{A^{\prime}} \nabla_{B}{ }^{B^{\prime}} h_{C D) A^{\prime} B^{\prime}}+\frac{1}{4} g^{e f} h_{e f} \Psi_{A B C D}
$$

Remark 24. We note that the spinor variational operator $\vartheta$ introduced in [Y] leads to a minus sign of the trace term in (4.1). Since the linearized metrics generated from a scalar potential are trace-free, i.e. $g^{\text {ef }} h_{e f}=0$, see (4.10) for details, the result would be the same for $\vartheta \Psi_{A B C D}$. However, since we are only interested in the ASD Weyl curvature here, there is no need to introduce the additional structures involving the $\vartheta$ variation.

To state the underlying operator identity of Wald's method, we begin with

Definition 25. Let $x_{a b}$ be a (possibly complex) symmetric 2-tensor, $\Phi$ be a complex scalar field of GHP weight $\{4,0\}$ and denote $l^{a} l^{b} x_{a b}$ by $x_{l l}$ etc. for tetrad components. Define the differential operators $\mathcal{S}, \mathcal{E}, \mathcal{O}, \mathcal{T}$ by

$$
\begin{aligned}
& \mathcal{S}\left(x_{a b}\right):=\left(\check{\partial}-4 \tau-\bar{\tau}^{\prime}\right)\left((b-2 \bar{\rho}) x_{l m}-\left(\check{\partial}-\bar{\tau}^{\prime}\right) x_{l l}\right) \\
& +(b-4 \rho-\bar{\rho})\left(\bar{\sigma}^{\prime} x_{l l}+\left(\check{\partial}-2 \bar{\tau}^{\prime}\right) x_{l m}-(p-\bar{\rho}) x_{m m}\right), \\
& \mathcal{E}(x)_{a b}:=\left(-\frac{1}{2} \square+6 \Lambda\right) x_{a b}-\frac{1}{2} \nabla_{a} \nabla_{b}\left(g^{c d} x_{c d}\right)+\nabla^{c} \nabla_{(a} x_{b) c}-\frac{1}{2} g_{a b}\left(\nabla^{c} \nabla^{d} x_{c d}-\square\left(g^{c d} x_{c d}\right)\right), \\
& \mathcal{O}(\Phi):=2\left[(b-4 \rho-\bar{\rho})\left(b^{\prime}-\rho^{\prime}\right)-\left(ð-4 \tau-\bar{\tau}^{\prime}\right)\left(\varpi^{\prime}-\tau^{\prime}\right)-3 \Psi_{2}\right] \Phi, \\
& \mathcal{T}\left(x_{a b}\right):=\frac{1}{2}\left(\left(\check{\partial}-2 \bar{\tau}^{\prime}\right) \partial-\bar{\sigma}^{\prime}(b-2 \bar{\rho})-2\left(b \bar{\sigma}^{\prime}\right)\right) x_{l l}+\frac{1}{2}(b-2 \bar{\rho}) p x_{m m} \\
& -\left((b-2 \bar{\rho})\left(\widetilde{\partial}-\bar{\tau}^{\prime}\right)-(\widetilde{\partial})\right) x_{l m} \text {. }
\end{aligned}
$$

Remark 26. (1) Note that $\mathcal{E}$ defined in $(4.2 \mathrm{~b})$ is the linearized Einstein operator plus a cosmological term. This operator is formally self adjoint,

$$
\mathcal{E}^{\dagger}=\mathcal{E}
$$

(2) Acting on a linearized metric $h_{a b}$, the operator $\mathcal{T}$ defined in (4.2d) yields the 0-component of the linearized anti-self dual Weyl curvature (4.1),

$$
\mathcal{T}\left(h_{a b}\right)=o^{A} o^{B} o^{C} o^{D} \dot{\Psi}_{A B C D}=\dot{\Psi}_{0} .
$$

(3) For any $h_{a b}$ such that $\mathcal{E}(h)_{a b}=0$, a decoupled wave-like (Teukolsky) equation is given by

$$
\mathcal{O T}\left(h_{a b}\right)=\mathcal{O}\left(\dot{\Psi}_{0}\right)=0 .
$$

Theorem 27 (Wald [29]). On Einstein spacetimes of Petrov type II with repeated principal spinor $o^{A}$, the operators of definition 25 satisfy the identity

$$
\mathcal{S E}=\mathcal{O} \mathcal{T} \text {. }
$$

Thus, in particular, if $\chi$ is a complex scalar field of GHP weight $\{-4,0\}$ solving

$$
\mathcal{O}^{\dagger} \chi=0,
$$

then the complex metric $h_{a b}=\mathcal{S}^{\dagger}(\chi)_{a b}$ solves the linearized Einstein equation

$$
\mathcal{E}(h)_{a b}=\mathcal{E}\left(\mathcal{S}^{\dagger}(\chi)\right)_{a b}=0 .
$$

In 29] the general idea was outlined and applied for Petrov type D, see [20] form of $\mathcal{S}$ is given on Petrov type II. The scalar field $\chi$ is called Debye potential and (4.7) is the Debye equation.

Lemma 28. Let $\chi$ be a complex scalar field of GHP weight $\{-4,0\}$.

- The operators adjoint to $\mathcal{S}$ and $\mathcal{O}$ are given by

$$
\begin{aligned}
& \mathcal{S}^{\dagger}(\chi)_{a b}=\left(l_{(a} m_{b)}(b-\rho+\bar{\rho})-l_{a} l_{b}(ð-\tau)\right)(\check{\partial}+3 \tau) \chi \\
&+\left(l_{(a} m_{b)}\left(ð-\tau+\bar{\tau}^{\prime}\right)-l_{a} l_{b} \bar{\sigma}^{\prime}-m_{a} m_{b}(b-\rho)\right)(b+3 \rho) \chi, \\
& \mathcal{O}^{\dagger} \chi=2\left(\left(p^{\prime}-\bar{\rho}^{\prime}\right)(b+3 \rho)-\left(\check{\partial}^{\prime}-\bar{\tau}\right)(ð+3 \tau)-3 \Psi_{2}\right) \chi .
\end{aligned}
$$

\footnotetext{
${ }^{7}$ It seems that there is a factor 4 missing in the operator identity in that reference.
} 
- If $\chi$ solves the Debye equation (4.7), then the complex metric $h_{a b}=\mathcal{S}^{\dagger}(\chi)_{a b}$ solving the linearized Einstein equation is given by

$$
h_{a b}=h_{n n} l_{a} l_{b}-2 h_{n \bar{m}} l_{(a} m_{b)}+h_{\bar{m} \bar{m}} m_{a} m_{b},
$$

with components

$$
\begin{aligned}
& h_{n n}=-(ð+2 \tau) \partial \chi-\bar{\sigma}^{\prime} b \chi, \\
& h_{n \bar{m}}=-(b+\rho) \partial \chi-\left(\tau+\bar{\tau}^{\prime}\right) p \chi, \\
& h_{\bar{m} \bar{m}}=-(b+2 \rho) b \chi .
\end{aligned}
$$

Proof. The formal adjoints of the GHP operators are given by

$$
\mathrm{p}^{\dagger}=-\mathrm{p}+\rho+\bar{\rho}, \quad \mathrm{p}^{\prime \dagger}=-\mathrm{p}^{\prime}+\rho^{\prime}+\bar{\rho}^{\prime}, \quad \mathrm{\partial}^{\dagger}=-\not{\partial}+\tau+\bar{\tau}^{\prime}, \quad \mathrm{\partial}^{\prime \dagger}=-\check{\partial}^{\prime}+\tau^{\prime}+\bar{\tau},
$$

see e.g. [1] for details. Using this, together with the rule $(A B)^{\dagger}=B^{\dagger} A^{\dagger}$ for compositions, to compute the adjoint of (4.2a) and (4.2C) leads to (4.9).

The equations (4.11) are the simplified components of (4.9a), using the commutator

$$
[\check{\partial}, \mathrm{b}] \chi=\left(\bar{\tau}^{\prime} \mathrm{p}-\bar{\rho} \check{\partial}\right) \chi,
$$

and Ricci identities,

$$
\mathrm{p} \rho=\rho^{2}, \quad \partial \tau=\tau^{2}-\bar{\sigma}^{\prime} \rho, \quad \mathrm{p} \tau=\rho\left(\tau-\bar{\tau}^{\prime}\right), \quad \partial \rho=\tau(\rho-\bar{\rho}),
$$

valid on type II Einstein spacetimes with repeated principal spinor $o^{A}$.

Before proceeding to the proof of Theorem 2, we need to introduce some additional identities and operators. First, similarly to Definition 10, we introduce:

Definition 29. Let $\left(o^{A}, \iota^{A}\right)$ be a spin dyad, $\Theta_{A A^{\prime}}$ the associated GHP connection, and $f_{A A^{\prime}}$ the 1 -form (2.6c). We define

$$
\begin{array}{ll}
\tilde{\Theta}_{A^{\prime}}:=O^{A} \Theta_{A A^{\prime}}, & \Theta_{A^{\prime}}:=\iota^{A} \Theta_{A A^{\prime}}, \\
\tilde{f}_{A^{\prime}}:=O^{A} f_{A A^{\prime}}, & f_{A^{\prime}}:=\iota^{A} f_{A A^{\prime}}, \\
& \sigma_{A^{\prime}}^{\prime}:=\iota^{A} \iota^{B} \nabla_{A A^{\prime} \iota_{B} .}
\end{array}
$$

Proposition 30. If $o^{A}$ is $S F R$, then

$$
\tilde{\Theta}_{A^{\prime}} O^{B}=0, \quad \Theta_{A^{\prime}} O^{B}=-\tilde{f}_{A^{\prime}} \iota^{B}, \quad \tilde{\Theta}_{A^{\prime}} \iota^{B}=-f_{A^{\prime}} O^{B}, \quad \Theta_{A^{\prime}} \iota^{B}=\sigma_{A^{\prime}}^{\prime} o^{B} .
$$

Remark 31. If $o^{A}$ is SFR and a repeated principal spinor, using (3.5) we note that $\tilde{f}_{A^{\prime}}$ can be written as

$$
\tilde{f}_{A^{\prime}}=\tilde{\Theta}_{A^{\prime}} \log \phi .
$$

Since in this section we are interested in the Einstein case, we can use (3.6) and replace $\phi=\Psi_{2}^{1 / 3}$.

Proposition 32. Let $\left(\mathcal{M}, g_{a b}\right)$ be a type II Einstein spacetime with repeated principal spinor $o^{A}$. On spinors of GHP weight $\{p, 0\}$ with an arbitrary number of primed indices the operators in (4.15) satisfy the following commutator relations,

$$
\begin{aligned}
& {\left[\tilde{\Theta}_{A^{\prime}}, \tilde{\Theta}_{B^{\prime}}\right]=0,} \\
& {\left[\tilde{\Theta}_{A^{\prime}}, \Theta_{B^{\prime}}\right]=\epsilon_{A^{\prime} B^{\prime}} \frac{p}{2}\left(2 \tilde{\Theta}_{C^{\prime}} f^{C^{\prime}}-\tilde{f}_{C^{\prime}} f^{C^{\prime}}+6 \Lambda\right)+\square_{A^{\prime} B^{\prime}}^{\Theta}-f_{A^{\prime}} \tilde{\Theta}_{B^{\prime}}+\tilde{f}_{B^{\prime}} \Theta_{A^{\prime}},} \\
& {\left[\Theta_{A^{\prime}}, \Theta_{B^{\prime}}\right]=\epsilon_{A^{\prime} B^{\prime}}\left(\frac{p}{2}\left(\Theta_{C^{\prime}} f^{C^{\prime}}-\tilde{\Theta}_{C^{\prime}} \sigma^{\prime C^{\prime}}-\tilde{f}_{C^{\prime}} \sigma^{\prime C^{\prime}}\right)-\sigma^{\prime C^{\prime}} \tilde{\Theta}_{C^{\prime}}\right) .}
\end{aligned}
$$

Proof. We prove (4.20a) explicitly, the other equations follow analogously. In a general spacetime, using definition (4.15) and acting on an arbitrary spinor $\varphi_{F \ldots F^{\prime} \ldots}^{E} \ldots E^{\prime} \ldots$ with GHP weight $\{p, q\}$ we get

$$
\left[\tilde{\Theta}_{A^{\prime}}, \tilde{\Theta}_{B^{\prime}}\right] \varphi_{F \ldots F^{\prime} \ldots}^{E \ldots E^{\prime} \ldots}=\bar{\epsilon}_{A^{\prime} B^{\prime}}\left(o^{A} o^{B} \nabla_{A} C^{C^{\prime}} o_{B}\right) \iota^{C} \Theta_{C C^{\prime}} \varphi_{F \ldots F^{\prime} \ldots}^{E \ldots}+o^{A} o^{B}\left[\Theta_{A A^{\prime}}, \Theta_{B B^{\prime}}\right] \varphi_{F \ldots F^{\prime}}^{E \ldots E^{\prime} \ldots} \text {. }
$$

For the second term in the right, using GHP notation we find

$$
\begin{aligned}
o^{A} o^{B}\left[\Theta_{A A^{\prime}}, \Theta_{B B^{\prime}}\right] \varphi_{F \ldots F^{\prime} \ldots}^{E \ldots E^{\prime} \ldots}= & \bar{\epsilon}_{A^{\prime} B^{\prime}} o^{A} o^{B} \Theta_{A C^{\prime}} \Theta_{B} C^{C^{\prime}} \varphi_{F \ldots F^{\prime} \ldots}^{E \ldots E^{\prime} \ldots} \\
= & \bar{\epsilon}_{A^{\prime} B^{\prime}} o^{A} o^{B} \square_{A B} \varphi_{F \ldots F^{\prime} \ldots}^{E \ldots E^{\prime} \ldots} \\
& +\left(p\left(-\Psi_{1}+\sigma \tau^{\prime}-\kappa \rho^{\prime}\right)+q\left(-\Phi_{01}+\bar{\tau}^{\prime} \bar{\rho}-\bar{\kappa} \bar{\sigma}^{\prime}\right)\right) \varphi_{F \ldots F^{\prime} \ldots}^{E \ldots E^{\prime} \ldots}
\end{aligned}
$$


where $\square_{A B}=\nabla_{A^{\prime}(A} \nabla_{B)} A^{\prime}$ is the usual spinor curvature operator [18, Eq. (4.9.2)]. Suppose now that the spacetime is Einstein and of Petrov type II. Restricting the identity (4.22) to primed spinor fields $\varphi_{F^{\prime}}^{E^{\prime} \ldots}$ with GHP weight $\{p, 0\}$, we get that each of the terms in the right hand side vanishes: $\square_{A B} \varphi_{F^{\prime} \ldots}^{E^{\prime} \cdots}=0$ because this only involves contractions with $\Phi_{A B A^{\prime} B^{\prime}}$, which vanishes because of the Einstein condition; the term with $q$ vanishes because we are restricting to weight $\{p, 0\}$; and the term with $p$ vanishes because the vacuum type II condition implies $\kappa=\sigma=\Psi_{1}=0$. Furthermore, from this last condition we get $o^{A} o^{B} \nabla_{A} C^{\prime} o_{B}=0$ (see (3.1)), therefore (4.21) vanishes identically and we get the result (4.20a).

Remark 33. Since $\left[\tilde{\Theta}_{A^{\prime}}, \tilde{\Theta}_{B^{\prime}}\right]=2 \tilde{\Theta}_{\left[A^{\prime}\right.} \tilde{\Theta}_{\left.B^{\prime}\right]}=-\bar{\epsilon}_{A^{\prime} B^{\prime}} \tilde{\Theta}^{C^{\prime}} \tilde{\Theta}_{C^{\prime}}$, the result (4.20a) can be equivalently stated as

$$
\tilde{\Theta}^{A^{\prime}} \tilde{\Theta}_{A^{\prime}} \varphi_{F^{\prime} \cdots}^{E^{\prime} \cdots}=0
$$

for any primed spinor $\varphi_{F^{\prime} \ldots}^{E^{\prime} \ldots}$ (or scalar) with weight $\{p, 0\}$. This identity will be useful below.

Proposition 34. Let $\tilde{\Theta}_{A^{\prime}}$ be as in (4.15), and let $\phi$ be given by (3.6). The linearized metric (4.10) can be expressed as

where

$$
h_{A A^{\prime} B B^{\prime}}=o_{A} O_{B} X_{A^{\prime} B^{\prime}}
$$

$$
X_{A^{\prime} B^{\prime}}=-\phi^{-2} \tilde{\Theta}_{\left(A^{\prime}\right.}\left(\phi^{2} \tilde{\Theta}_{\left.B^{\prime}\right)} \chi\right) .
$$

Proof. This can be shown by direct comparison with (4.10)-4.11). First, from (4.24) we see that the only non-trivial components are

$$
\begin{aligned}
& X_{1^{\prime} 1^{\prime}}=-\iota^{A} \bar{\iota}^{A^{\prime}} \iota^{B} \bar{\iota}^{B^{\prime}} h_{A A^{\prime} B B^{\prime}}=-n^{a} n^{b} h_{a b}=-h_{n n}, \\
& X_{0^{\prime} 1^{\prime}}=-\iota^{A} \bar{\iota}^{A^{\prime}} \iota^{B} \bar{o}^{B^{\prime}} h_{A A^{\prime} B B^{\prime}}=-n^{a} \bar{m}^{b} h_{a b}=-h_{n \bar{m}}, \\
& X_{0^{\prime} 0^{\prime}}=-\iota^{A} \bar{o}^{A^{\prime}} \iota^{B} \bar{o}^{B^{\prime}} h_{A A^{\prime} B B^{\prime}}=-\bar{m}^{a} \bar{m}^{b} h_{a b}=-h_{\bar{m} \bar{m}},
\end{aligned}
$$

so the general structure (4.10) is recovered. Now we check that these components coincide with (4.11). To do this, we write $X_{A^{\prime} B^{\prime}}$ as

$$
X_{A^{\prime} B^{\prime}}=-\tilde{\Theta}_{A^{\prime}} \tilde{\Theta}_{B^{\prime}} \chi-2\left(\tilde{\Theta}_{\left(A^{\prime}\right.} \log \phi\right)\left(\tilde{\Theta}_{\left.B^{\prime}\right)} \chi\right) .
$$

Next, we project this expression over the primed spin dyad $\left(\bar{o}^{A^{\prime}}, \bar{\iota}^{A^{\prime}}\right)$ to compute the components (4.26). We will need the following identities:

$$
\mathrm{p} \log \phi=\rho, \quad \partial \log \phi=\tau,
$$

which follow from the fact that $\phi=\Psi_{2}^{1 / 3}$ (eq. (3.6) ) together with the Bianchi identities for a Petrov type II spacetime. Then we have, for example,

$$
\begin{aligned}
X_{1^{\prime} 1^{\prime}} & =-\bar{\iota}^{A^{\prime}} \bar{\iota}^{B^{\prime}} \tilde{\Theta}_{A^{\prime}} \tilde{\Theta}_{B^{\prime}} \chi-2 ð \log \phi \partial \chi \\
& =-\partial \partial \chi+\left(\partial \bar{\iota}^{B^{\prime}}\right) \tilde{\Theta}_{B^{\prime}} \chi-2 \tau \partial \chi \\
& =-(\partial+2 \tau) \partial \chi-\bar{\sigma}^{\prime} \mathrm{p} \chi
\end{aligned}
$$

where we used $\partial \bar{\iota}^{B^{\prime}}=-\bar{\sigma}^{\prime} \bar{o}^{B^{\prime}}$, see [18, Eq. (4.12.28)]. The other components can be computed along the same line.

Proposition 35. Let $\left(\mathcal{M}, g_{a b}\right)$ be a type II Einstein spacetime with repeated principal spinor $o^{A}$. The Ricci, Bianchi and commutator identities yield

$$
\begin{aligned}
\tilde{\Theta}_{A^{\prime}} \tilde{f}_{B^{\prime}} & =\tilde{f}_{A^{\prime}} \tilde{f}_{B^{\prime}}, \\
\tilde{\Theta}^{A^{\prime}} f_{A^{\prime}} & =\Psi_{2}+2 \Lambda, \\
\Theta^{A^{\prime}} \tilde{f}_{A^{\prime}} & =-\Psi_{2}-2 \Lambda, \\
\tilde{\Theta}_{A^{\prime}} \Psi_{2} & =3 \tilde{f}_{A^{\prime}} \Psi_{2}, \\
\tilde{\Theta}_{A^{\prime}} \tilde{\Theta}_{B^{\prime}} \phi^{-1} & =0,
\end{aligned}
$$

with the scalar field $\phi$ defined in (3.6).

Proof. These equations can be checked by GHP expansion using for example [18, Section 4.12]. 
The main result of this section is given in lemma 37 below, from which theorem 2 follows. The proof of lemma 37 involves long computations and we transfer some intermediate steps into the following:

Proposition 36. The Debye equation in this formulation is given by

$$
\mathcal{O}^{\dagger} \chi=2 \Theta^{A^{\prime}}\left(\tilde{\Theta}_{A^{\prime}}+3 \tilde{f}_{A^{\prime}}\right) \chi-6 \Psi_{2} \chi
$$

Repeated application of commutators on $\chi$ of weight $\{-4,0\}$ leads to

$$
\begin{aligned}
\tilde{\Theta}_{A^{\prime}} \Theta_{B^{\prime}} \tilde{\Theta}^{B^{\prime}} \tilde{\Theta}^{A^{\prime}} \chi= & -\tilde{f}^{A^{\prime}} \tilde{f}^{B^{\prime}} \Theta_{B^{\prime}} \tilde{\Theta}_{A^{\prime}} \chi+3 \tilde{f}^{A^{\prime}} \Lambda \tilde{\Theta}_{A^{\prime}} \chi-\frac{15}{2} \tilde{f}^{A^{\prime}} \Psi_{2} \tilde{\Theta}_{A^{\prime}} \chi-\frac{3}{2} f^{A^{\prime}} \tilde{f}^{B^{\prime}} \tilde{\Theta}_{A^{\prime}} \tilde{\Theta}_{B^{\prime}} \chi \\
& -3 f^{A^{\prime}} \tilde{f}_{A^{\prime}} \tilde{f}^{B^{\prime}} \tilde{\Theta}_{B^{\prime}} \chi+\tilde{f}^{A^{\prime}} \tilde{\Theta}_{B^{\prime}} \Theta^{B^{\prime}} \tilde{\Theta}_{A^{\prime}} \chi-\frac{3}{2} f^{A^{\prime}} \tilde{f}^{B^{\prime}} \tilde{\Theta}_{B^{\prime}} \tilde{\Theta}_{A^{\prime}} \chi \\
& +\frac{3}{2} \tilde{f}^{A^{\prime}} \tilde{\Theta}_{A^{\prime}} f_{B^{\prime}} \tilde{\Theta}^{B^{\prime}} \chi-\frac{3}{2} \tilde{f}^{A^{\prime}} \tilde{\Theta}_{B^{\prime}} f_{A^{\prime}} \tilde{\Theta}^{B^{\prime}} \chi
\end{aligned}
$$

$$
\begin{aligned}
\tilde{\Theta}_{B^{\prime}} \Theta_{A^{\prime}} \tilde{\Theta}^{B^{\prime}} \tilde{\Theta}^{A^{\prime}} \chi= & -\tilde{f}^{A^{\prime}} \tilde{f}^{B^{\prime}} \Theta_{B^{\prime}} \tilde{\Theta}_{A^{\prime}} \chi+3 \tilde{f}^{A^{\prime}} \Lambda \tilde{\Theta}_{A^{\prime}} \chi-\frac{15}{2} \tilde{f}^{A^{\prime}} \Psi_{2} \tilde{\Theta}_{A^{\prime}} \chi-\frac{3}{2} f^{A^{\prime}} \tilde{f}^{B^{\prime}} \tilde{\Theta}_{A^{\prime}} \tilde{\Theta}_{B^{\prime}} \chi \\
& -3 f^{A^{\prime}} \tilde{f}_{A^{\prime}} \tilde{f}^{B^{\prime}} \tilde{\Theta}_{B^{\prime}} \chi+\tilde{f}^{A^{\prime}} \tilde{\Theta}_{B^{\prime}} \Theta^{B^{\prime}} \tilde{\Theta}_{A^{\prime}} \chi-\frac{3}{2} f^{A^{\prime}} \tilde{f}^{B^{\prime}} \tilde{\Theta}_{B^{\prime}} \tilde{\Theta}_{A^{\prime}} \chi \\
& +\frac{3}{2} \tilde{f}^{A^{\prime}} \tilde{\Theta}_{A^{\prime}} f_{B^{\prime}} \tilde{\Theta}^{B^{\prime}} \chi-\frac{3}{2} \tilde{f}^{A^{\prime}} \tilde{\Theta}_{B^{\prime}} f_{A^{\prime}} \tilde{\Theta}^{B^{\prime}} \chi
\end{aligned}
$$

$$
\begin{aligned}
\Theta_{B^{\prime}} \Theta_{A^{\prime}} \tilde{\Theta}^{B^{\prime}} \tilde{\Theta}^{A^{\prime}} \chi= & -9 \chi f^{A^{\prime}} \tilde{f}_{A^{\prime}} \Lambda+18 \chi \Lambda^{2}-\frac{3}{4} f^{A^{\prime}} \tilde{f}_{A^{\prime}} \mathcal{O}^{\dagger} \chi+\frac{9}{2} \Lambda \mathcal{O}^{\dagger} \chi+\frac{1}{4} \mathcal{O}^{\dagger} \mathcal{O}^{\dagger} \chi-9 \chi f^{A^{\prime}} \tilde{f}_{A^{\prime}} \Psi_{2} \\
& -36 \chi \Lambda \Psi_{2}+\frac{3}{2} \mathcal{O}^{\dagger} \chi \Psi_{2}-36 \chi \Psi_{2}^{2}+3 \tilde{f}^{A^{\prime}} \Lambda \Theta_{A^{\prime}} \chi-30 \tilde{f}^{A^{\prime}} \Psi_{2} \Theta_{A^{\prime}} \chi \\
& +\frac{3}{2} \tilde{f}^{A^{\prime}} \Theta_{A^{\prime}} \mathcal{O}^{\dagger} \chi-18 \chi \tilde{f}^{A^{\prime}} \Theta_{A^{\prime}} \Psi_{2}-3 \Lambda \Theta_{A^{\prime}} \tilde{\Theta}^{A^{\prime}} \chi-6 \Psi_{2} \Theta_{A^{\prime}} \tilde{\Theta}^{A^{\prime}} \chi \\
& -\frac{3}{2} f^{A^{\prime}} \tilde{f}^{B^{\prime}} \Theta_{A^{\prime}} \tilde{\Theta}_{B^{\prime}} \chi-\frac{9}{2} f^{A^{\prime}} \tilde{f}_{A^{\prime}} \tilde{f}^{B^{\prime}} \Theta_{B^{\prime}} \chi-3 \tilde{f}^{A^{\prime}} \tilde{f}^{B^{\prime}} \Theta_{B^{\prime}} \Theta_{A^{\prime}} \chi \\
& +\tilde{f}^{A^{\prime}} \Theta_{B^{\prime}} \Theta^{B^{\prime}} \tilde{\Theta}_{A^{\prime}} \chi-\frac{3}{2} f^{A^{\prime}} \tilde{f}^{B^{\prime}} \Theta_{B^{\prime}} \tilde{\Theta}_{A^{\prime}} \chi-3 \tilde{f}^{A^{\prime}} \Theta_{B^{\prime}} \tilde{\Theta}^{B^{\prime}} \Theta_{A^{\prime}} \chi \\
& +3 \tilde{f}^{A^{\prime}} \Theta_{A^{\prime}} \tilde{f}_{B^{\prime}} \Theta^{B^{\prime}} \chi-\Theta_{B^{\prime}} \tilde{\Theta}_{A^{\prime}} \chi \Theta^{B^{\prime}} \tilde{f}^{A^{\prime}}-3 f^{A^{\prime}} \Lambda \tilde{\Theta}_{A^{\prime}} \chi-\frac{3}{2} f^{A^{\prime}} \Psi_{2} \tilde{\Theta}_{A^{\prime}} \chi \\
& +3 \Theta^{A^{\prime}} \Psi_{2} \tilde{\Theta}_{A^{\prime}} \chi-\frac{3}{2} \tilde{f}^{A^{\prime}} \Theta_{B^{\prime}} f^{B^{\prime}} \tilde{\Theta}_{A^{\prime}} \chi-\frac{3}{2} \tilde{f}^{A^{\prime}} \Theta_{A^{\prime}} f^{B^{\prime}} \tilde{\Theta}_{B^{\prime}} \chi \\
& -\frac{3}{2} f^{A^{\prime}} \Theta_{A^{\prime}} \tilde{f}^{B^{\prime}} \tilde{\Theta}_{B^{\prime}} \chi+\frac{3}{2} \tilde{f}^{A^{\prime}} \Theta^{B^{\prime}} f_{A^{\prime}} \tilde{\Theta}_{B^{\prime}} \chi-\frac{3}{2} f^{A^{\prime}} \Theta^{B^{\prime}} \tilde{f}_{A^{\prime}} \tilde{\Theta}_{B^{\prime}} \chi \\
& +3 \Theta^{B^{\prime}} \tilde{f}^{A^{\prime}} \tilde{\Theta}_{B^{\prime}} \Theta_{A^{\prime}} \chi .
\end{aligned}
$$

$$
\begin{aligned}
\Theta_{B^{\prime}} \Theta_{A^{\prime}} \tilde{\Theta}^{B^{\prime}} \chi= & -6 \Lambda \Theta_{A^{\prime}} \chi-6 \Psi_{2} \Theta_{A^{\prime}} \chi-\frac{1}{2} \Theta_{A^{\prime}} \mathcal{O}^{\dagger} \chi-6 \chi \Theta_{A^{\prime}} \Psi_{2}+3 \tilde{f}_{B^{\prime}} \Theta_{A^{\prime}} \Theta^{B^{\prime}} \chi \\
& +3 \Theta_{A^{\prime}} \tilde{f}_{B^{\prime}} \Theta^{B^{\prime}} \chi-\frac{3}{2} \tilde{f}_{B^{\prime}} \sigma^{B^{\prime}} \tilde{\Theta}_{A^{\prime}} \chi-\frac{3}{2} \Theta_{B^{\prime}} f^{B^{\prime}} \tilde{\Theta}_{A^{\prime}} \chi+\frac{3}{2} \tilde{\Theta}_{A^{\prime}} \chi \tilde{\Theta}_{B^{\prime}} \sigma^{B^{\prime}} \\
& -\sigma^{B^{\prime}} \tilde{\Theta}_{B^{\prime}} \tilde{\Theta}_{A^{\prime}} \chi
\end{aligned}
$$

$$
\begin{aligned}
\Theta_{A^{\prime}} \tilde{\Theta}^{A^{\prime}} \Theta^{B^{\prime}} \chi= & -4 \chi f^{B^{\prime}} \Lambda-2 \chi f^{B^{\prime}} \Psi_{2}+4 \chi \tilde{f}^{A^{\prime}} \tilde{f}^{B^{\prime}} \sigma_{A^{\prime}}^{\prime}-2 f^{B^{\prime}} \tilde{f}^{A^{\prime}} \Theta_{A^{\prime}} \chi-2 f^{A^{\prime}} \tilde{f}^{B^{\prime}} \Theta_{A^{\prime}} \chi \\
& -6 \chi \tilde{f}^{B^{\prime}} \Theta_{A^{\prime}} f^{A^{\prime}}-2 \chi \tilde{f}^{A^{\prime}} \Theta_{A^{\prime}} f^{B^{\prime}}-2 \chi f^{A^{\prime}} \Theta_{A^{\prime}} \tilde{f}^{B^{\prime}}-\tilde{f}^{B^{\prime}} \Theta_{A^{\prime}} \Theta^{A^{\prime}} \chi-f^{A^{\prime}} \Theta_{A^{\prime}} \tilde{\Theta}^{B^{\prime}} \chi \\
& +\Theta \tilde{f}^{\prime} \tilde{f}^{B^{\prime}} \Theta^{A^{\prime}} \chi-2 f^{A^{\prime}} \tilde{f}_{A^{\prime}} \Theta^{B^{\prime}} \chi-2 \Lambda \Theta^{B^{\prime}} \chi-10 \Psi_{2} \Theta^{B^{\prime}} \chi+2 \chi \tilde{f}^{A^{\prime}} \Theta^{B^{\prime}} f_{A^{\prime}} \\
& -2 \chi f^{A^{\prime}} \Theta^{B^{\prime}} \tilde{f}_{A^{\prime}}+3 \Theta^{A^{\prime}} \chi \Theta^{B^{\prime}} \tilde{f}_{A^{\prime}}-\frac{1}{2} \Theta^{B^{\prime}} O^{\dagger} \chi-10 \chi \Theta^{B^{\prime}} \Psi_{2}-3 \tilde{f}^{A^{\prime}} \Theta^{B^{\prime}} \Theta_{A^{\prime}} \chi \\
& -2 \tilde{f}^{B^{\prime}} \sigma^{\prime A^{\prime}} \tilde{\Theta}_{A^{\prime}} \chi+4 \chi \tilde{f}^{B^{\prime}} \tilde{\Theta}_{A^{\prime}} \sigma^{A^{\prime}}-\sigma^{A^{\prime}} \tilde{\Theta}_{A^{\prime}} \tilde{\Theta}^{B^{\prime}} \chi+\frac{3}{2} \tilde{f}^{A^{\prime}} \sigma_{A^{\prime}}^{\prime} \tilde{\Theta}^{B^{\prime}} \chi \\
& -\frac{5}{2} \Theta_{A^{\prime}} f^{A^{\prime}} \tilde{\Theta}^{B^{\prime}} \chi+\frac{3}{2} \tilde{\Theta}_{A^{\prime}} \sigma^{A^{\prime}} \tilde{\Theta}^{B^{\prime}} \chi .
\end{aligned}
$$

$$
\Theta_{B^{\prime}} \Theta^{B^{\prime}} \tilde{\Theta}_{A^{\prime}} \chi=\frac{1}{2}\left(-3 \tilde{f}_{B^{\prime}} \sigma^{\prime B^{\prime}} \tilde{\Theta}_{A^{\prime}} \chi-3 \Theta_{B^{\prime}} f^{B^{\prime}} \tilde{\Theta}_{A^{\prime}} \chi+3 \tilde{\Theta}_{A^{\prime}} \chi \tilde{\Theta}_{B^{\prime}} \sigma^{\prime B^{\prime}}-2 \sigma^{\prime B^{\prime}} \tilde{\Theta}_{B^{\prime}} \tilde{\Theta}_{A^{\prime}} \chi\right)
$$

Proof. The identities are verified by direct computation using the projected operators $\Theta_{A^{\prime}}, \tilde{\Theta}_{A^{\prime}}$ defined in (4.15) and their commutator properties (4.20). The Debye equation (4.31) can be seen to coincide with (4.9b) by GHP expansion. 
Lemma 37. The ASD curvature components of a metric of the form (4.10), (4.11) are given by

$$
\begin{aligned}
& \dot{\Psi}_{0}=0 \\
& \dot{\Psi}_{1}=0 \\
& \dot{\Psi}_{2}=0 \\
& \dot{\Psi}_{3}=-\frac{1}{4}(\tau p-\rho ð) \mathcal{O}^{\dagger} \chi \\
& \dot{\Psi}_{4}=-\left(\Psi_{2}^{4 / 3} \xi^{a} \Theta_{a}+3 \Psi_{2}^{2}+6 \Psi_{2} \Lambda\right) \chi-\left(\frac{1}{8} \mathcal{O}^{\dagger}-\rho p^{\prime}+\tau \varpi^{\prime}+2 \Psi_{2}+\frac{5}{2} \Lambda\right) \mathcal{O}^{\dagger} \chi .
\end{aligned}
$$

Proof. The result is verified by direct computation using the projected operators $\Theta_{A^{\prime}}, \tilde{\Theta}_{A^{\prime}}$ defined in (4.15) and their commutator properties (4.20). The first step is to derive an appropriate form for the components of the linearized ASD Weyl curvature spinor (4.1). Replacing (4.24) in (4.1) and using (4.15), (4.16), (4.18), we find

$$
\begin{aligned}
& \dot{\Psi}_{0}=0 \\
& \dot{\Psi}_{1}=0 \\
& \dot{\Psi}_{2}=\frac{1}{12}\left(\tilde{\Theta}_{B^{\prime}}+2 \tilde{f}_{B^{\prime}}\right)\left(\tilde{\Theta}_{A^{\prime}}+2 \tilde{f}_{A^{\prime}}\right) X^{A^{\prime} B^{\prime}} \\
& \dot{\Psi}_{3}=\frac{1}{8}\left(\left(\Theta_{B^{\prime}}+f_{B^{\prime}}\right)\left(\tilde{\Theta}_{A^{\prime}}+2 \tilde{f}_{A^{\prime}}\right)+\left(\tilde{\Theta}_{B^{\prime}}+3 \tilde{f}_{B^{\prime}}\right) \Theta_{A^{\prime}}\right) X^{A^{\prime} B^{\prime}}, \\
& \dot{\Psi}_{4}=\frac{1}{2}\left(\Theta_{B^{\prime}} \Theta_{A^{\prime}}-\sigma_{B^{\prime}}^{\prime}\left(\tilde{\Theta}_{A^{\prime}}+2 \tilde{f}_{A^{\prime}}\right)\right) X^{A^{\prime} B^{\prime}} .
\end{aligned}
$$

So the first two equations, (4.39a) and (4.39b), follow from the algebraic structure of (4.25). For $\dot{\Psi}_{2}$, rewrite the operator in (4.39c) using (4.19) and insert (4.25),

$$
\dot{\Psi}_{2}=\frac{1}{12} \phi^{-2} \tilde{\Theta}_{B^{\prime}} \tilde{\Theta}_{A^{\prime}}\left(\phi^{2} X^{A^{\prime} B^{\prime}}\right)=\frac{1}{12} \phi^{-2} \tilde{\Theta}_{B^{\prime}} \tilde{\Theta}_{A^{\prime}} \tilde{\Theta}^{A^{\prime}}\left(\phi^{2} \tilde{\Theta}^{B^{\prime}} \chi\right)=0,
$$

where the last step follows from (4.23). Next we compute $\dot{\psi}_{3}$ by first inserting (4.25) into (4.39d) and expanding out,

$$
\begin{aligned}
& \dot{\Psi}_{3}=-\frac{1}{8} \tilde{f}^{A^{\prime}} \Theta_{A^{\prime}} \tilde{\Theta}_{B^{\prime}} \tilde{\Theta}^{B^{\prime}} \chi-\frac{1}{16} \Theta_{A^{\prime}} \tilde{\Theta}_{B^{\prime}} \tilde{\Theta}^{B^{\prime}} \tilde{\Theta}^{A^{\prime}} \chi+\frac{5}{8} \tilde{f}^{A^{\prime}} \tilde{f}^{B^{\prime}} \Theta_{B^{\prime}} \tilde{\Theta}_{A^{\prime}} \chi+\frac{3}{16} \tilde{f}^{A^{\prime}} \Theta_{B^{\prime}} \tilde{\Theta}_{A^{\prime}} \tilde{\Theta}^{B^{\prime}} \chi \\
& -\frac{1}{16} \Theta_{B^{\prime}} \tilde{\Theta}_{A^{\prime}} \tilde{\Theta}^{B^{\prime}} \tilde{\Theta}^{A^{\prime}} \chi+\frac{5}{16} \tilde{f}^{A^{\prime}} \Theta_{B^{\prime}} \tilde{\Theta}^{B^{\prime}} \tilde{\Theta}_{A^{\prime}} \chi+\frac{5}{8} \tilde{f}^{A^{\prime}} \Theta_{B^{\prime}} \tilde{f}^{B^{\prime}} \tilde{\Theta}_{A^{\prime}} \chi+\frac{1}{4} \Theta^{B^{\prime}} \tilde{\Theta}^{A^{\prime}} \chi \tilde{\Theta}_{A^{\prime}} \tilde{f}_{B^{\prime}} \\
& -\frac{1}{8} \tilde{f}^{A^{\prime}} \tilde{\Theta}_{A^{\prime}} \Theta_{B^{\prime}} \tilde{\Theta}^{B^{\prime}} \chi-\frac{1}{16} \tilde{\Theta}_{A^{\prime}} \Theta_{B^{\prime}} \tilde{\Theta}^{B^{\prime}} \tilde{\Theta}^{A^{\prime}} \chi-\frac{1}{8} f^{A^{\prime}} \tilde{f}^{B^{\prime}} \tilde{\Theta}_{A^{\prime}} \tilde{\Theta}_{B^{\prime}} \chi+\frac{1}{8} \Theta^{B^{\prime}} \tilde{f}^{A^{\prime}} \tilde{\Theta}_{A^{\prime}} \tilde{\Theta}_{B^{\prime}} \chi \\
& -\frac{1}{8} \tilde{\Theta}_{A^{\prime}} \Theta_{B^{\prime}} \tilde{f}^{B^{\prime}} \tilde{\Theta}^{A^{\prime}} \chi-\frac{1}{4} f^{A^{\prime}} \tilde{f}_{A^{\prime}} \tilde{f}^{B^{\prime}} \tilde{\Theta}_{B^{\prime}} \chi+\frac{1}{4} \tilde{f}^{A^{\prime}} \Theta_{A^{\prime}} \tilde{f}^{B^{\prime}} \tilde{\Theta}_{B^{\prime}} \chi+\frac{1}{8} \Theta_{A^{\prime}} \tilde{\Theta}^{B^{\prime}} \tilde{f}^{A^{\prime}} \tilde{\Theta}_{B^{\prime}} \chi \\
& -\frac{3}{8} \tilde{f}^{A^{\prime}} \Theta^{B^{\prime}} \tilde{f}_{A^{\prime}} \tilde{\Theta}_{B^{\prime}} \chi+\frac{1}{8} \Theta^{B^{\prime}} \tilde{\Theta}_{A^{\prime}} \tilde{f}^{A^{\prime}} \tilde{\Theta}_{B^{\prime}} \chi-\frac{1}{4} \Theta_{A^{\prime}} \tilde{\Theta}^{A^{\prime}} \chi \tilde{\Theta}_{B^{\prime}} \tilde{f}^{B^{\prime}}+\frac{1}{8} f^{A^{\prime}} \tilde{\Theta}_{A^{\prime}} \chi \tilde{\Theta}_{B^{\prime}}, \tilde{f}^{B^{\prime}} \\
& -\frac{1}{8} \tilde{\Theta}^{A^{\prime}} \chi \tilde{\Theta}_{B^{\prime}} \Theta_{A^{\prime}} \tilde{f}^{B^{\prime}}-\frac{1}{8} \tilde{f}^{A^{\prime}} \tilde{\Theta}_{B^{\prime}} \Theta_{A^{\prime}} \tilde{\Theta}^{B^{\prime}} \chi-\frac{1}{16} \tilde{\Theta}_{B^{\prime}} \Theta_{A^{\prime}} \tilde{\Theta}^{B^{\prime}} \tilde{\Theta}^{A^{\prime}} \chi-\frac{1}{8} \Theta^{B^{\prime}} \tilde{f}^{A^{\prime}} \tilde{\Theta}_{B^{\prime}} \tilde{\Theta}_{A^{\prime}} \chi \\
& +\frac{1}{16} f^{A^{\prime}} \tilde{\Theta}_{B^{\prime}} \tilde{\Theta}_{A^{\prime}} \tilde{\Theta}^{B^{\prime}} \chi+\frac{1}{8} f^{A^{\prime}} \tilde{f}_{A^{\prime}} \tilde{\Theta}_{B^{\prime}} \tilde{\Theta}^{B^{\prime}} \chi-\frac{1}{4} \Theta_{A^{\prime}} \tilde{f}^{A^{\prime}} \tilde{\Theta}_{B^{\prime}} \tilde{\Theta}^{B^{\prime}} \chi+\frac{1}{16} f^{A^{\prime}} \tilde{\Theta}_{B^{\prime}} \tilde{\Theta}^{B^{\prime}} \tilde{\Theta}_{A^{\prime}} \chi \\
& +\frac{1}{8} f^{A^{\prime}} \tilde{\Theta}_{B^{\prime}} \tilde{f}_{A^{\prime}} \tilde{\Theta}^{B^{\prime}} \chi \text {. }
\end{aligned}
$$

Using (4.30) and (4.20a) yields

$$
\begin{aligned}
\dot{\Psi}_{3}= & \frac{7}{8} \tilde{f}^{A^{\prime}} \tilde{f}^{B^{\prime}} \Theta_{B^{\prime}} \tilde{\Theta}_{A^{\prime}} \chi+\frac{1}{2} \tilde{f}^{A^{\prime}} \Theta_{B^{\prime}} \tilde{\Theta}^{B^{\prime}} \tilde{\Theta}_{A^{\prime}} \chi+\frac{3}{2} \tilde{f}^{A^{\prime}} \Lambda \tilde{\Theta}_{A^{\prime}} \chi+\frac{9}{8} \tilde{f}^{A^{\prime}} \Psi_{2} \tilde{\Theta}_{A^{\prime}} \chi-\frac{1}{8} \tilde{f}^{A^{\prime}} \tilde{\Theta}_{A^{\prime}} \Theta_{B^{\prime}} \tilde{\Theta}^{B^{\prime}} \chi \\
& -\frac{1}{16} \tilde{\Theta}_{A^{\prime}} \Theta_{B^{\prime}} \tilde{\Theta}^{B^{\prime}} \tilde{\Theta}^{A^{\prime}} \chi-\frac{3}{8} f^{A^{\prime}} \tilde{f}_{A^{\prime}} \tilde{f}^{B^{\prime}} \tilde{\Theta}_{B^{\prime}} \chi+\frac{3}{8} \tilde{f}^{A^{\prime}} \Theta_{A^{\prime}} \tilde{f}^{B^{\prime}} \tilde{\Theta}_{B^{\prime}} \chi-\frac{3}{8} \tilde{f}^{A^{\prime}} \Theta^{B^{\prime}} \tilde{f}_{A^{\prime}} \tilde{\Theta}_{B^{\prime}} \chi \\
& -\frac{1}{8} \tilde{\Theta}^{A^{\prime}} \chi \tilde{\Theta}_{B^{\prime}} \Theta_{A^{\prime}} \tilde{f}^{B^{\prime}}-\frac{1}{8} \tilde{f}^{A^{\prime}} \tilde{\Theta}_{B^{\prime}} \Theta_{A^{\prime}} \tilde{\Theta}^{B^{\prime}} \chi-\frac{1}{16} \tilde{\Theta}_{B^{\prime}} \Theta_{A^{\prime}} \tilde{\Theta}^{B^{\prime}} \tilde{\Theta}^{A^{\prime}} \chi-\frac{1}{8} f^{A^{\prime}} \tilde{f}^{B^{\prime}} \tilde{\Theta}_{B^{\prime}} \tilde{\Theta}_{A^{\prime}} \chi
\end{aligned}
$$

To eliminate 4th order terms, we use (4.32) and (4.33), leading to

$$
\begin{aligned}
\dot{\Psi}_{3}= & \tilde{f}^{A^{\prime}} \tilde{f}^{B^{\prime}} \Theta_{B^{\prime}} \tilde{\Theta}_{A^{\prime}} \chi+\frac{1}{2} \tilde{f}^{A^{\prime}} \Theta_{B^{\prime}} \tilde{\Theta}^{B^{\prime}} \tilde{\Theta}_{A^{\prime}} \chi+\frac{9}{8} \tilde{f}^{A^{\prime}} \Lambda \tilde{\Theta}_{A^{\prime}} \chi+\frac{33}{16} \tilde{f}^{A^{\prime}} \Psi_{2} \tilde{\Theta}_{A^{\prime}} \chi-\frac{1}{8} \tilde{f}^{A^{\prime}} \tilde{\Theta}_{A^{\prime}} \Theta_{B^{\prime}} \tilde{\Theta}^{B^{\prime}} \chi \\
& +\frac{3}{16} f^{A^{\prime}} \tilde{f}^{B^{\prime}} \tilde{\Theta}_{A^{\prime}} \tilde{\Theta}_{B^{\prime}} \chi+\frac{3}{8} \tilde{f}^{A^{\prime}} \Theta_{A^{\prime}} \tilde{f}^{B^{\prime}} \tilde{\Theta}_{B^{\prime}} \chi-\frac{3}{8} \tilde{f}^{A^{\prime}} \Theta^{B^{\prime}} \tilde{f}_{A^{\prime}} \tilde{\Theta}_{B^{\prime}} \chi-\frac{1}{8} \tilde{\Theta}^{A^{\prime}} \chi \tilde{\Theta}_{B^{\prime}} \Theta_{A^{\prime}} \tilde{f}^{B^{\prime}} \\
& -\frac{1}{8} \tilde{f}^{A^{\prime}} \tilde{\Theta}_{B^{\prime}} \Theta_{A^{\prime}} \tilde{\Theta}^{B^{\prime}} \chi-\frac{1}{8} \tilde{f}^{A^{\prime}} \tilde{\Theta}_{B^{\prime}} \Theta^{B^{\prime}} \tilde{\Theta}_{A^{\prime}} \chi+\frac{1}{16} f^{A^{\prime}} \tilde{f}^{B^{\prime}} \tilde{\Theta}_{B^{\prime}} \tilde{\Theta}_{A^{\prime}} \chi-\frac{3}{16} \tilde{f}^{A^{\prime}} \tilde{\Theta}_{A^{\prime}} f_{B^{\prime}} \tilde{\Theta}^{B^{\prime}} \chi \\
& +\frac{3}{16} \tilde{f}^{A^{\prime}} \tilde{\Theta}_{B^{\prime}} f_{A^{\prime}} \tilde{\Theta}^{B^{\prime}} \chi .
\end{aligned}
$$


Using the commutators (4.20a), (4.20b) and the Debye equation (4.31) together with (4.30) takes care of third order terms,

$$
\begin{aligned}
\dot{\Psi}_{3}= & -\frac{13}{8} \tilde{f}^{A^{\prime}} \Lambda \tilde{\Theta}_{A^{\prime}} \chi-\frac{13}{16} \tilde{f}^{A^{\prime}} \Psi_{2} \tilde{\Theta}_{A^{\prime}} \chi-\frac{1}{4} \tilde{f}^{A^{\prime}} \tilde{\Theta}_{A^{\prime}} \mathcal{O}^{\dagger} \chi+\frac{3}{8} \tilde{f}^{A^{\prime}} \Theta_{A^{\prime}} \tilde{f}^{B^{\prime}} \tilde{\Theta}_{B^{\prime}} \chi-\frac{3}{8} \tilde{f}^{A^{\prime}} \Theta^{B^{\prime}} \tilde{f}_{A^{\prime}} \tilde{\Theta}_{B^{\prime}} \chi \\
& +\frac{7}{16} \tilde{f}^{A^{\prime}} \tilde{\Theta}_{A^{\prime}} f_{B^{\prime}} \tilde{\Theta}^{B^{\prime}} \chi-\frac{7}{16} \tilde{f}^{A^{\prime}} \tilde{\Theta}_{B^{\prime}} f_{A^{\prime}} \tilde{\Theta}^{B^{\prime}} \chi .
\end{aligned}
$$

Finally, the irreducible decompositions

$$
\begin{aligned}
& \tilde{\Theta}_{A^{\prime}} f_{B^{\prime}}=\tilde{\Theta}_{\left(A^{\prime}\right.} f_{\left.B^{\prime}\right)}+\frac{1}{2} \bar{\epsilon}_{A^{\prime} B^{\prime}} \tilde{\Theta}_{C^{\prime}} f^{C^{\prime}}, \\
& \Theta_{A^{\prime}} \tilde{f}_{B^{\prime}}=\Theta_{\left(A^{\prime}\right.} \tilde{f}_{\left.B^{\prime}\right)}+\frac{1}{2} \bar{\epsilon}_{A^{\prime} B^{\prime}} \Theta_{C^{\prime}} \tilde{f}^{C^{\prime}},
\end{aligned}
$$

lead to

$$
\dot{\Psi}_{3}=-\frac{1}{4} \tilde{f}^{A^{\prime}} \tilde{\Theta}_{A^{\prime}} \mathcal{O}^{\dagger} \chi
$$

which gives (4.38d) by GHP expanding $\tilde{f}^{A^{\prime}} \tilde{\Theta}_{A^{\prime}}$.

To compute $\dot{\Psi}_{4}$, insert (4.25) into (4.39e) and use the identity (4.34) together with the Debye equation (4.31) and (4.30) leading to

$$
\begin{aligned}
& \dot{\Psi}_{4}=\frac{9}{2} \chi f^{A^{\prime}} \tilde{f}_{A^{\prime}} \Lambda-18 \chi \Lambda^{2}+\frac{3}{8} f^{A^{\prime}} \tilde{f}_{A^{\prime}} \mathcal{O}^{\dagger} \chi-2 \Lambda \mathcal{O}^{\dagger} \chi-\frac{1}{8} \mathcal{O}^{\dagger} \mathcal{O}^{\dagger} \chi+\frac{9}{2} \chi f^{A^{\prime}} \tilde{f}_{A^{\prime}} \Psi_{2}+6 \chi \Lambda \Psi_{2} \\
& -\frac{7}{4} \mathcal{O}^{\dagger} \chi \Psi_{2}+\frac{15}{2} \chi \Psi_{2}^{2}+\frac{21}{2} \tilde{f}^{A^{\prime}} \Psi_{2} \Theta_{A^{\prime}} \chi+6 \chi \Lambda \Theta_{A^{\prime}} \tilde{f}^{A^{\prime}}-\frac{3}{2} \chi \Psi_{2} \Theta_{A^{\prime}} \tilde{f}^{A^{\prime}}-\frac{1}{2} \tilde{f}^{A^{\prime}} \Theta_{A^{\prime}} \mathcal{O}^{\dagger} \chi \\
& +\frac{21}{2} \chi \tilde{f}^{A^{\prime}} \Theta_{A^{\prime}} \Psi_{2}+\frac{3}{2} \chi \tilde{f}^{A^{\prime}} \Theta_{A^{\prime}} \Theta_{B^{\prime}} \tilde{f}^{B^{\prime}}+\frac{3}{4} f^{A^{\prime}} \tilde{f}^{B^{\prime}} \Theta_{A^{\prime}} \tilde{\Theta}_{B^{\prime}} \chi+\frac{9}{4} f^{A^{\prime}} \tilde{f}_{A^{\prime}} \tilde{f}^{B^{\prime}} \Theta_{B^{\prime}} \chi \\
& +\frac{3}{2} \tilde{f}^{A^{\prime}} \Theta_{A^{\prime}} \chi \Theta_{B^{\prime}} \tilde{f}^{B^{\prime}}+3 \tilde{f}^{A^{\prime}} \tilde{f}^{B^{\prime}} \Theta_{B^{\prime}} \Theta_{A^{\prime}} \chi-\frac{1}{2} \tilde{f}^{A^{\prime}} \Theta_{B^{\prime}} \Theta_{A^{\prime}} \tilde{\Theta}^{B^{\prime}} \chi-\frac{1}{2} \tilde{f}^{A^{\prime}} \Theta_{B^{\prime}} \Theta^{B^{\prime}} \tilde{\Theta}_{A^{\prime}} \chi \\
& +\frac{3}{4} f^{A^{\prime}} \tilde{f}^{B^{\prime}} \Theta_{B^{\prime}} \tilde{\Theta}_{A^{\prime}} \chi+\frac{3}{2} \tilde{f}^{A^{\prime}} \Theta_{B^{\prime}} \tilde{\Theta}^{B^{\prime}} \Theta_{A^{\prime}} \chi-3 \tilde{f}^{A^{\prime}} \Theta_{A^{\prime}} \tilde{f}_{B^{\prime}} \Theta^{B^{\prime}} \chi+\Theta_{A^{\prime}} \tilde{\Theta}_{B^{\prime}} \chi \Theta^{B^{\prime}} \tilde{f}^{A^{\prime}} \\
& +\frac{1}{2} \Theta_{B^{\prime}} \tilde{\Theta}_{A^{\prime}} \chi \Theta^{B^{\prime}} \tilde{f}^{A^{\prime}}+\frac{3}{2} f^{A^{\prime}} \Lambda \tilde{\Theta}_{A^{\prime}} \chi+\frac{3}{4} f^{A^{\prime}} \Psi_{2} \tilde{\Theta}_{A^{\prime}} \chi-\Theta^{A^{\prime}} \Psi_{2} \tilde{\Theta}_{A^{\prime}} \chi+\frac{3}{4} \tilde{f}^{A^{\prime}} \Theta_{B^{\prime}} f^{B^{\prime}} \tilde{\Theta}_{A^{\prime}} \chi \\
& -\frac{3}{2} \tilde{f}^{A^{\prime}} \tilde{f}^{B^{\prime}} \sigma_{A^{\prime}}^{\prime} \tilde{\Theta}_{B^{\prime}} \chi+\frac{3}{4} \tilde{f}^{A^{\prime}} \Theta_{A^{\prime}} f^{B^{\prime}} \tilde{\Theta}_{B^{\prime}} \chi+\frac{3}{4} f^{A^{\prime}} \Theta_{A^{\prime}} \tilde{f}^{B^{\prime}} \tilde{\Theta}_{B^{\prime}} \chi+\frac{1}{2} \Theta_{A^{\prime}} \Theta^{B^{\prime}} \tilde{f}^{A^{\prime}} \tilde{\Theta}_{B^{\prime}} \chi \\
& -\frac{3}{4} \tilde{f}^{A^{\prime}} \Theta^{B^{\prime}} f_{A^{\prime}} \tilde{\Theta}_{B^{\prime}} \chi+\frac{3}{4} f^{A^{\prime}} \Theta^{B^{\prime}} \tilde{f}_{A^{\prime}} \tilde{\Theta}_{B^{\prime}} \chi-\frac{3}{2} \Theta^{B^{\prime}} \tilde{f}^{A^{\prime}} \tilde{\Theta}_{B^{\prime}} \Theta_{A^{\prime}} \chi+\frac{1}{2} \tilde{f}^{A^{\prime}} \sigma^{\prime B^{\prime}} \tilde{\Theta}_{B^{\prime}} \tilde{\Theta}_{A^{\prime}} \chi \\
& -\frac{1}{4} \sigma^{\prime A^{\prime}} \tilde{\Theta}_{B^{\prime}} \tilde{\Theta}_{A^{\prime}} \tilde{\Theta}^{B^{\prime}} \chi \text {. }
\end{aligned}
$$

To convert third order terms we use identities (4.35), (4.36), 4.37). After a commutator (4.20b) and (4.30) is used, we have

$$
\begin{aligned}
\dot{\Psi}_{4}= & \frac{9}{2} \chi f^{A^{\prime}} \tilde{f}_{A^{\prime}} \Lambda+6 \chi \Lambda^{2}+\frac{3}{8} f^{A^{\prime}} \tilde{f}_{A^{\prime}} \mathcal{O}^{\dagger} \chi-2 \Lambda \mathcal{O}^{\dagger} \chi-\frac{1}{8} \mathcal{O}^{\dagger} \mathcal{O}^{\dagger} \chi+\frac{9}{2} \chi f^{A^{\prime}} \tilde{f}_{A^{\prime}} \Psi_{2}+3 \chi \Lambda \Psi_{2} \\
& -\frac{7}{4} \mathcal{O}^{\dagger} \chi \Psi_{2}+3 \tilde{f}^{A^{\prime}} \Lambda \Theta_{A^{\prime}} \chi-\tilde{f}^{A^{\prime}} \Theta_{A^{\prime}} \mathcal{O}^{\dagger} \chi-\frac{3}{4} f^{A^{\prime}} \tilde{f}^{B^{\prime}} \Theta_{A^{\prime}} \tilde{\Theta}_{B^{\prime}} \chi+\frac{9}{4} f^{A^{\prime}} \tilde{f}_{A^{\prime}} \tilde{f}^{B^{\prime}} \Theta_{B^{\prime}} \chi \\
& +\frac{3}{4} f^{A^{\prime}} \tilde{f}^{B^{\prime}} \Theta_{B^{\prime}} \tilde{\Theta}_{A^{\prime}} \chi-\frac{1}{2} \Theta_{A^{\prime}} \tilde{\Theta}_{B^{\prime}} \chi \Theta^{B^{\prime}} \tilde{f}^{A^{\prime}}+\frac{1}{2} \Theta_{B^{\prime}} \tilde{\Theta}_{A^{\prime}} \chi \Theta^{B^{\prime}} \tilde{f}^{A^{\prime}}+\frac{3}{2} f^{A^{\prime}} \Lambda \tilde{\Theta}_{A^{\prime}} \chi \\
& +\frac{3}{4} f^{A^{\prime}} \Psi_{2} \tilde{\Theta}_{A^{\prime}} \chi-\Theta^{A^{\prime}} \Psi_{2} \tilde{\Theta}_{A^{\prime}} \chi-\frac{3}{2} \tilde{f}^{A^{\prime}} \Theta_{B^{\prime}} f^{B^{\prime}} \tilde{\Theta}_{A^{\prime}} \chi-\frac{3}{4} \tilde{f}^{A^{\prime}} \tilde{f}^{B^{\prime}} \sigma_{A^{\prime}}^{\prime} \tilde{\Theta}_{B^{\prime}} \chi \\
& +\frac{3}{4} \tilde{f}^{A^{\prime}} \Theta_{A^{\prime}} f^{B^{\prime}} \tilde{\Theta}_{B^{\prime}} \chi-\frac{3}{4} f^{A^{\prime}} \Theta_{A^{\prime}} \tilde{f}^{B^{\prime}} \tilde{\Theta}_{B^{\prime}} \chi+\frac{1}{2} \Theta_{A^{\prime}} \Theta^{B^{\prime}} \tilde{f}^{A^{\prime}} \tilde{\Theta}_{B^{\prime}} \chi-\frac{3}{4} \tilde{f}^{A^{\prime}} \Theta^{B^{\prime}} f_{A^{\prime}} \tilde{\Theta}_{B^{\prime}} \chi \\
& +\frac{3}{4} f^{A^{\prime}} \Theta^{B^{\prime}} \tilde{f}_{A^{\prime}} \tilde{\Theta}_{B^{\prime}} \chi+\frac{3}{4} \tilde{f}^{A^{\prime}} \tilde{\Theta}_{A^{\prime}} \chi \tilde{\Theta}_{B^{\prime}} \sigma^{B^{\prime}}-\frac{1}{4} \sigma^{A^{\prime}} \tilde{\Theta}_{B^{\prime}} \tilde{\Theta}_{A^{\prime}} \tilde{\Theta}^{B^{\prime}} \chi
\end{aligned}
$$

Now, the commutator (4.20a) together with the irreducible decomposition

$$
\Theta_{A^{\prime}} \tilde{\Theta}_{B^{\prime}} \chi=\Theta_{\left(A^{\prime}\right.} \tilde{\Theta}_{\left.B^{\prime}\right)} \chi+\frac{1}{2} \bar{\epsilon}_{A^{\prime} B^{\prime}} \Theta_{C^{\prime}} \tilde{\Theta}^{C^{\prime}} \chi,
$$

and (4.31), 4.30) yields

$$
\begin{aligned}
\dot{\Psi}_{4}= & -\frac{5}{2} \Lambda \mathcal{O}^{\dagger} \chi-\frac{1}{8} \mathcal{O}^{\dagger} \mathcal{O}^{\dagger} \chi-6 \chi \Lambda \Psi_{2}-2 \mathcal{O}^{\dagger} \chi \Psi_{2}-3 \chi \Psi_{2}^{2}-\frac{3}{2} \tilde{f}^{A^{\prime}} \Psi_{2} \Theta_{A^{\prime}} \chi-\tilde{f}^{A^{\prime}} \Theta_{A^{\prime}} \mathcal{O}^{\dagger} \chi \\
& +\frac{3}{2} f^{A^{\prime}} \Lambda \tilde{\Theta}_{A^{\prime}} \chi+\frac{3}{4} f^{A^{\prime}} \Psi_{2} \tilde{\Theta}_{A^{\prime}} \chi-\frac{1}{2} \Theta^{A^{\prime}} \Psi_{2} \tilde{\Theta}_{A^{\prime}} \chi-\frac{5}{4} \tilde{f}^{A^{\prime}} \Theta_{B^{\prime}} f^{B^{\prime}} \tilde{\Theta}_{A^{\prime}} \chi \\
& -\frac{1}{2} \tilde{f}^{A^{\prime}} \tilde{f}^{B^{\prime}} \sigma_{A^{\prime}}^{\prime} \tilde{\Theta}_{B^{\prime}} \chi+\frac{3}{4} \tilde{f}^{A^{\prime}} \Theta_{A^{\prime}} f^{B^{\prime}} \tilde{\Theta}_{B^{\prime}} \chi-\frac{3}{4} f^{A^{\prime}} \Theta_{A^{\prime}} \tilde{f}^{B^{\prime}} \tilde{\Theta}_{B^{\prime}} \chi-\frac{3}{4} \tilde{f}^{A^{\prime}} \Theta^{B^{\prime}} f_{A^{\prime}} \tilde{\Theta}_{B^{\prime}} \chi \\
& +\frac{3}{4} f^{A^{\prime}} \Theta^{B^{\prime}} \tilde{f}_{A^{\prime}} \tilde{\Theta}_{B^{\prime}} \chi+\frac{1}{2} \tilde{f}^{A^{\prime}} \tilde{\Theta}_{A^{\prime}} \chi \tilde{\Theta}_{B^{\prime}} \sigma^{B^{\prime}} .
\end{aligned}
$$

The Ricci identity

$$
2 \Psi_{3}-\tilde{f}_{B^{\prime}} \sigma^{\prime B^{\prime}}+\Theta_{A^{\prime}} f^{A^{\prime}}-\tilde{\Theta}_{B^{\prime}} \sigma^{B^{\prime}}=0 .
$$


and the irreducible decompositions (4.45b) and

$$
\Theta_{A^{\prime}} f_{B^{\prime}}=\Theta_{\left(A^{\prime}\right.} f_{\left.B^{\prime}\right)}+\frac{1}{2} \bar{\epsilon}_{A^{\prime} B^{\prime}} \Theta_{C^{\prime}} f^{C^{\prime}}
$$

lead to

$$
\begin{aligned}
\dot{\Psi}_{4}= & -\frac{5}{2} \Lambda \mathcal{O}^{\dagger} \chi-\frac{1}{8} \mathcal{O}^{\dagger} \mathcal{O}^{\dagger} \chi-6 \chi \Lambda \Psi_{2}-2 \mathcal{O}^{\dagger} \chi \Psi_{2}-3 \chi \Psi_{2}^{2}-\frac{3}{2} \tilde{f}^{A^{\prime}} \Psi_{2} \Theta_{A^{\prime}} \chi-\tilde{f}^{A^{\prime}} \Theta_{A^{\prime}} \mathcal{O}^{\dagger} \chi \\
& +\tilde{f}^{A^{\prime}} \Psi_{3} \tilde{\Theta}_{A^{\prime}} \chi-\frac{1}{2} \Theta^{A^{\prime}} \Psi_{2} \tilde{\Theta}_{A^{\prime}} \chi .
\end{aligned}
$$

To bring it into the final form we use the Bianchi identity

$$
-3 f_{A^{\prime}} \Psi_{2}+2 \tilde{f}_{A^{\prime}} \Psi_{3}+\Theta_{A^{\prime}} \Psi_{2}-\tilde{\Theta}_{A^{\prime}} \Psi_{3}=0,
$$

resulting in

$$
\begin{aligned}
\dot{\Psi}_{4}= & -\frac{3}{2} \Psi_{2}\left(f^{A^{\prime}} \tilde{\Theta}_{A^{\prime}}+\tilde{f}^{A^{\prime}} \Theta_{A^{\prime}}+2 \Psi_{2}+4 \Lambda\right) \chi+\frac{1}{2}\left(\tilde{\Theta}_{A^{\prime}}-4 \tilde{f}_{A^{\prime}}\right) \Psi_{3} \tilde{\Theta}^{A^{\prime}} \chi \\
& -\left(\frac{1}{8} \mathcal{O}^{\dagger}+\tilde{f}^{A^{\prime}} \Theta_{A^{\prime}}+2 \Psi_{2}+\frac{5}{2} \Lambda\right) \mathcal{O}^{\dagger} \chi .
\end{aligned}
$$

GHP expansion of $\tilde{f}_{A^{\prime}}, \Theta_{A^{\prime}}, \tilde{\Theta}_{A^{\prime}}$ leads to

$$
\begin{aligned}
\dot{\Psi}_{4}= & -\frac{3}{2} \Psi_{2}\left(\rho^{\prime} \mathrm{p}-\rho \mathrm{p}^{\prime}+\tau \partial^{\prime}-\tau^{\prime} \partial+2 \Psi_{2}+4 \Lambda\right) \chi+\frac{1}{2}\left((\mathrm{~b}-4 \rho) \Psi_{3}\right) \partial \chi-\frac{1}{2}\left((ð-4 \tau) \Psi_{3}\right) \mathrm{p} \chi \\
& -\left(\frac{1}{8} \mathcal{O}^{\dagger}-\rho \mathrm{p}^{\prime}+\tau \check{\partial}^{\prime}+2 \Psi_{2}+\frac{5}{2} \Lambda\right) \mathcal{O}^{\dagger} \chi .
\end{aligned}
$$

Comparison to the projected Killing vector defined in (3.26) shows (4.38e).

Proof of Theorem [. The result follows from Lemma37by imposing the Debye equation (4.7).

Remark 38. In the special case of vacuum Petrov type $D$ and for tetrads invariant under $\xi^{a}$, (4.38el) reduces to $\dot{\Psi}_{4}=\xi^{a} \nabla_{a} \chi$, see [1].

Remark 39. Let us finally compare to three references closely related to the results of this section.

(1) In [15] Kegeles and Cohen discuss Debye potentials for algebraically special geometries. They restricted to vacuum Petrov type D for the derivation of the linearized Weyl spinor, see equation (5.28) in that reference. They reduced the ASD Weyl curvature to type $N$ and also the $\dot{\Psi}_{4}$ to first order.

(2) In [20], Stewart derived the linearized connection and curvature components for vacuum type II perturbations in terms of a Debye potential. However, the result was not fully simplified, see equation (4.27) of that reference, so that the type $N$ property could not be observed. The result was also presented in terms of a real metric, which means that all terms involving $\bar{\chi}$ correspond to self dual Weyl curvature, while $\chi$ terms belong to anti-self dual Weyl curvature.

It should also be noted that in general, linearized Dyad components differ from dyad components of the linearized field. In this paper $\dot{\Psi}_{i}$ refers to the latter, while Stewart used the linearized Newman-Penrose equations, i.e. the former. However, he made a special choice of linearized tetrad for which the two sets of linearized Weyl components coincide.

(3) In [14], Jeffryes discusses a reduction to scalar potentials for algebraically special solutions to the full non-linear Einstein-Yang-Mills equations. Further it is shown that, to linear order, this construction reduces to the Debye potential formulation. Remarkably, the ASD Weyl curvature can be simplified already on the non-linear level, so that Theorem 2 we discuss here follows from the linearized equations (8.75-77) of that reference.

Acknowledgements. This work started while the authors were in residence at Institut MittagLeffler in Djursholm, Sweden during the fall of 2019, supported by the Swedish Research Council under grant no. 2016-06596. SA thanks Thomas Bäckdahl for xAct support and Benjamin Jeffryes for comments on his preprint [14]. BA is supported by a postdoctoral fellowship from Conicet (Argentina). BFW acknowledges support from NSF grant PHY 1607323, sabbatical support from the University of Florida and the Observaroire de Paris at Meudon, and the Institut d'Astrophysique de Paris. 


\section{Appendix A. Robinson-Trautman metrics}

In 1968, Robinson and Trautman, 22], published a line element for which the vacuum Einstein equations reduce essentially to a non-linear fourth order equation for a real scalar function. It admits solutions of all Petrov types. Here we briefly review the reduction and also the explicit example of Petrov type II given in [22].

In coordinates $(u, r, \zeta, \bar{\zeta})$ and with real functions $H, P$, define the tetrad

$$
l^{a}=\left(\partial_{r}\right)^{a}, \quad n^{a}=\left(\partial_{u}\right)^{a}-H\left(\partial_{r}\right)^{a}, \quad m^{a}=\operatorname{Pr}^{-1}\left(\partial_{\zeta}\right)^{a}, \quad \bar{m}^{a}=\operatorname{Pr}^{-1}\left(\partial_{\bar{\zeta}}\right)^{a} .
$$

Due to the normalization $l^{a} n_{a}=1, m^{a} \bar{m}_{a}=-1$, the inverse is given by

$$
l_{a}=\mathrm{d} u_{a}, \quad n_{a}=\mathrm{d} r_{a}+H \mathrm{~d} u_{a}, \quad m_{a}=-r P^{-1} \mathrm{~d} \zeta_{a}, \quad \bar{m}_{a}=-r P^{-1} \mathrm{~d} \bar{\zeta}_{a},
$$

so that the metric $g_{a b}=2 l_{(a} n_{b)}-2 m_{(a} \bar{m}_{b)}$ is of the form

$$
g_{a b}=2 \mathrm{~d} r_{(a} \mathrm{d} u_{b)}+2 H \mathrm{~d} u_{a} \mathrm{~d} u_{b}-2 r^{2} P^{-2} \mathrm{~d} \zeta_{(a} \mathrm{d} \bar{\zeta}_{b)} .
$$

This metric is Ricci flat if $H$ is given by

$$
H=P^{2} \partial_{\zeta} \partial_{\bar{\zeta}} \log (P)-r \partial_{u} \log (P)-m r^{-1}, \quad \text { with } m=m(u),
$$

and $P$ being independent of $r$, solving

$$
P^{2} \partial_{\zeta} \partial_{\bar{\zeta}}\left(P^{2} \partial_{\zeta} \partial_{\bar{\zeta}} \log (P)\right)-\partial_{u} m+3 m \partial_{u} \log (P)=0 .
$$

For the connection and curvature we find

$$
\kappa=\sigma=\sigma^{\prime}=\tau=\tau^{\prime}=\epsilon=0, \quad R_{a b}=0, \quad \Psi_{0}=\Psi_{1}=0,
$$

in particular the metric is algebraically special. The non-vanishing spin coefficients are given by

$$
\begin{aligned}
& \kappa^{\prime}=-\operatorname{Pr}^{-1} \partial_{\bar{\zeta}} H, \\
& \rho=-r^{-1}, \\
& \rho^{\prime}=H r^{-1} \partial_{u} \log (P), \\
& \gamma=\frac{1}{2} \partial_{r} H, \\
& \alpha=\frac{1}{2} r^{-1} \partial_{\bar{\zeta}} P, \\
& \beta=-\frac{1}{2} r^{-1} \partial_{\zeta} P,
\end{aligned}
$$

with $H$ given in (A.4) and the remaining Weyl components are of the form

$$
\begin{aligned}
& \Psi_{2}=-m r^{-3}, \quad \Psi_{3}=-P r^{-2} \partial_{\bar{\zeta}}\left(P^{2} \partial_{\zeta} \partial_{\bar{\zeta}} \log (P)\right), \\
& \Psi_{4}=r^{-2} \partial_{\bar{\zeta}}\left(P^{2} \partial_{\bar{\zeta}}\left(P^{2} \partial_{\zeta} \partial_{\bar{\zeta}} \log (P)-r \partial_{u} \log (P)\right)\right) .
\end{aligned}
$$

An explicit example of a type II geometry, found in [22], is given by

$$
P=(\zeta+\bar{\zeta})^{3 / 2}, \quad m=\text { const. }
$$

leading to $H=-m / r-3(\zeta+\bar{\zeta}) / 2$ and to the metric

$$
g_{a b}=2 \mathrm{~d} r_{(a} \mathrm{d} u_{b)}-(3(\zeta+\bar{\zeta})+2 m / r) \mathrm{d} u_{a} \mathrm{~d} u_{b}-2 r^{2}(\zeta+\bar{\zeta})^{-3} \mathrm{~d} \zeta_{(a} \mathrm{d} \bar{\zeta}_{b)},
$$

see also [25, §28]. The spin coefficients reduce to

$$
\kappa^{\prime}=\frac{3(\zeta+\bar{\zeta})^{3 / 2}}{2 r}, \quad \rho=-\frac{1}{r}, \quad \rho^{\prime}=-\frac{m}{r^{2}}-\frac{3(\zeta+\bar{\zeta})}{2 r}, \quad \gamma=\frac{m}{2 r^{2}}, \quad \alpha=-\beta=\frac{3(\zeta+\bar{\zeta})^{1 / 2}}{4 r},
$$

and the curvature components are given by

$$
\Psi_{2}=-\frac{m}{r^{3}}, \quad \Psi_{3}=\frac{3(\zeta+\bar{\zeta})^{3 / 2}}{2 r^{2}}, \quad \Psi_{4}=-\frac{9(\zeta+\bar{\zeta})^{2}}{2 r^{2}}
$$

\section{REFERENCES}

[1] Steffen Aksteiner. Geometry and analysis in black hole spacetimes. PhD thesis, Gottfried Wilhelm Leibniz Universität Hannover, 2014. http://d-nb.info/1057896721.

[2] Steffen Aksteiner and Thomas Bäckdahl. SpinFrames, 2015-2018. SpinFrames.

[3] Steffen Aksteiner and Thomas Bäckdahl. Symmetries of linearized gravity from adjoint operators. Journal of Mathematical Physics, 60(8):082501, August 2019.

[4] Lars Andersson, Thomas Bäckdahl, and Pieter Blue. Second order symmetry operators. Class. Quant. Grav., 31:135015, 2014.

[5] Bernardo Araneda. Conformal invariance, complex structures and the Teukolsky connection. Classical and Quantum Gravity, 35(17):175001, September 2018. 
[6] Bernardo Araneda. Two-dimensional twistor manifolds and Teukolsky operators. Letters in Mathematical Physics, 110(10):2603-2638, June 2020.

[7] Thomas Bäckdahl and Juan A. Valiente Kroon. A formalism for the calculus of variations with spinors. Journal of Mathematical Physics, 57(2):022502, February 2016.

[8] Leor Barack and Paco Giudice. Time-domain metric reconstruction for self-force applications. Phys. Rev. D, 95(10):104033, 2017.

[9] P.L. Chrzanowski. Vector Potential and Metric Perturbations of a Rotating Black Hole. Phys. Rev. D, 11:2042-2062, 1975.

[10] Robert Geroch, Alan Held, and Roger Penrose. A space-time calculus based on pairs of null directions. Journal of Mathematical Physics, 14(7):874-881, 1973.

[11] Stephen R. Green, Stefan Hollands, and Peter Zimmerman. Teukolsky formalism for nonlinear Kerr perturbations. Class. Quant. Grav., 37(7):075001, 2020.

[12] Lane P Hughston and Paul Sommers. The symmetries of kerr black holes. Communications in Mathematical Physics, 33(2):129-133, 1973.

[13] Benjamin P. Jeffryes. Space-times with two-index Killing spinors. Proceedings of the Royal Society of London Series A, 392(1803):323-341, April 1984.

[14] Benjamin P. Jeffryes. Half-algebraically special space-times, field equations and potentials. Max Planck Institut fur Astrophysik Report, 263:12-101, January 1986.

[15] Lawrence S Kegeles and Jeffrey M Cohen. Constructive procedure for perturbations of spacetimes. Physical Review D, 19(6):1641, 1979.

[16] Carlos O. Lousto and Bernard F. Whiting. Reconstruction of black hole metric perturbations from Weyl curvature. Phys. Rev. D, 66:024026, 2002.

[17] Cesar Merlin, Amos Ori, Leor Barack, Adam Pound, and Maarten van de Meent. Completion of metric reconstruction for a particle orbiting a Kerr black hole. Phys. Rev. D, 94(10):104066, November 2016.

[18] Roger Penrose and Wolfgang Rindler. Spinors and space-time: Volume 1, Two-spinor calculus and relativistic fields, volume 1. Cambridge University Press, 1984.

[19] Roger Penrose and Wolfgang Rindler. Spinors and space-time: Volume 2, Spinor and twistor methods in space-time geometry, volume 2. Cambridge University Press, 1986.

[20] Kartik Prabhu and Robert M. Wald. Canonical energy and Hertz potentials for perturbations of Schwarzschild spacetime. Classical and Quantum Gravity, 35(23):235004, December 2018.

[21] Larry R. Price, Karthik Shankar, and Bernard F. Whiting. On the existence of radiation gauges in Petrov type II spacetimes. Classical and Quantum Gravity, 24(9):2367-2388, May 2007.

[22] I. Robinson and A. Trautman. Some Spherical Gravitational Waves in General Relativity. Proceedings of the Royal Society of London Series A, 265(1323):463-473, February 1962.

[23] Krzysztof Rózga. Real slices of complex space-time in general relativity. Reports on Mathematical Physics, 11(2):197-210, April 1977.

[24] R. Sachs. Gravitational Waves in General Relativity. VI. The Outgoing Radiation Condition. Proceedings of the Royal Society of London Series A, 264(1318):309-338, November 1961.

[25] Hans Stephani, Dietrich Kramer, Malcolm MacCallum, Cornelius Hoenselaers, and Eduard Herlt. Exact Solutions of Einstein's Field Equations. Cambridge Monographs on Mathematical Physics. Cambridge University Press, 2 edition, 2003.

[26] J. M. Stewart. Hertz-Bromwich-Debye-Whittaker-Penrose potentials in general relativity. Proceedings of the Royal Society of London Series A, 367(1731):527-538, September 1979.

[27] G. F. Torres Del Castillo. Gravitational perturbations of type-D vacuum space-times with cosmological constant. Journal of Mathematical Physics, 35(6):3051-3058, June 1994.

[28] G. F. Torres del Castillo and H. G. Solís-Rodríguez. Self-duality and gravitational perturbations. Journal of Mathematical Physics, 40(8):4099-4105, August 1999.

[29] Robert M. Wald. Construction of Solutions of Gravitational, Electromagnetic, Or Other Perturbation Equations from Solutions of Decoupled Equations. Phys. Rev. Lett., 41:203206, 1978.

[30] Martin Walker and Roger Penrose. On quadratic first integrals of the geodesic equations for type $\{22\}$ spacetimes. Communications in Mathematical Physics, 18(4):265-274, 1970.

[31] Nicholas Woodhouse. The Real Geometry of Complex Space-Times. International Journal of Theoretical Physics, 16(9):663-670, September 1977. 
Email address: steffen.aksteiner@aei.mpg.de

Albert Einstein Institute, Am Mühlenberg 1, D-14476 Potsdam, Germany

Email address: lars.andersson@aei.mpg.de

Albert Einstein Institute, Am Mühlenberg 1, D-14476 Potsdam, Germany

Email address: baraneda@f amaf.unc.edu.ar

Facultad de Matemática, Astronomía, Física y Computación, Universidad Nacional de Córdoba, instituto de Física Enrique Gaviola, COniCET, Ciudad Universitaria, (5000) Córdoba, Argentina

Email address: bernard@phys.ufl.edu

Department of Physics, University of Florida, 2001 Museum Road, Gainesville, FL 32611-8440, USA 